\title{
STOCHASTIC FORMS OF \\ FUNCTIONAL ISOPERIMETRIC \\ INEQUALITIES
}

\section{Jesus Rebollo Bueno}

A Dissertation presented to the Faculty of the Graduate School at the University of Missouri-Columbia in Partial Fulfillment of the Requirements for the Degree of

Doctor of Philosophy

Dr. Peter Pivovarov, Dissertation Supervisor

Department of Mathematics

University of Missouri-Columbia

USA

May of 2021 
(C)Copyright by Jesus Rebollo Bueno 2021 
The undersigned, appointed by the dean of the Graduate School, have examined the dissertation

\section{STOCHASTIC FORMS OF FUNCTIONAL ISOPERIMETRIC INEQUALITIES}

presented by Jesus Rebollo Bueno,

a candidate for the degree of doctor of philosophy, and hereby certify that, in their opinion, it is worthy of acceptance.

Peter Pivovarov, Ph.D.

David M. Kaplan , Ph.D.

Alexander Koldobsky, Ph.D.

Petros Valettas, Ph.D. 


\section{ACKNOWLEDGMENTS}

This dissertation, and more broadly my success in the PhD program, would not have been possible without the overwhelming support and eternal patience of my academic advisor, Peter Pivovarov, to whom I owe more than I can ever repay. I would like to extend my sincere gratitude to professors Alexander Koldobsky and Petros Valettas for offering numerous topics courses that I found very interesting.

I am also grateful to my mentor as an undergraduate and master's student, Rafael Villa Caro, for introducing me to convex geometry when no courses about the topic were offered, sparking my current research interests.

My friends and fellows in the PhD program also contributed heavily to my success; not only did countless conversations help enrich my understanding, but they also offered invaluable support at my arrival to a new country and culture. Among these I must thank, Georgios Dosidis, Dorsa Ghoreishi, Jose Luís Luna García, and Roberto Nuñez.

Por último, pero no menos importante, me gustaría agradecer a los miembros de mi familia. A mis padres, Jesús Rebollo Gil y Esperanza Bueno Hinojosa; por todo su apoyo, motivación, y ánimos a perseguir mis objetivos. A mi hermana, Sara Rebollo Bueno, por su bondad y cariño infinitos; no podría haber pedido una mejor. A mi hermano de diferentes padres, Álvaro Gómez Núñez, regalo irremplazable del universo. A mi compaẽra de vida, Kristal Marie Bas Sánchez, por mantenerme cuerdo y lleno de felicidad en los momentos difíciles. 


\section{CONTENTS}

Acknowledgments

Abstract $\quad$ v

1 Introduction 1

1.1 Sylvester's dual and planar inequalities . . . . . . . . . . . . . . 2

1.2 Stochastic Prékopa-Leinder via bodies of revolution . . . . . . . . 8

1.3 Stochastic Brunn's principle via epigraphs and hypographs . . . . . 10

2 Stochastic Reverse Isoperimetric Inequalities On The Plane 17

2.1 Definitions and notation . . . . . . . . . . . . 17

2.2 Dual version of Sylvester's functional . . . . . . . . . . . . . . 20

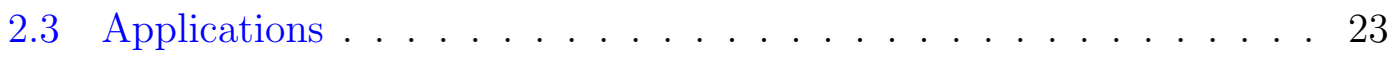

3 A Stochastic Prékopa-Leindler Inequality for Log-Concave Func$\begin{array}{ll}\text { tions } & 30\end{array}$

3.1 Definitions and notation . . . . . . . . . . . . 30

3.2 Approximation of log-concave functions . . . . . . . . . . . . . 31

3.3 Convex hull and $\mathcal{M}$-addition operations . . . . . . . . . . . 35

3.4 Multiple integral rearrangement inequalities . . . . . . . . . 36

3.5 Proofs of stochastic Prékopa-Leindler and Groemer inequalities . . . 40

4 Stochastic Forms of Brunn's Principle $\quad 47$

4.1 Rinott's approach to s-concave functions . . . . . . . . . . . . 47

$4.2 \mathcal{M}$-addition and multiple integral rearrangement inequalities . . . . 50 
4.3 Shadow systems of $s$-concave functions . . . . . . . . . . . 51

4.4 Random epigraphs and hypographs . . . . . . . . . . . . . 56

4.5 Proof of Stochastic Brunn's principle . . . . . . . . . . . . . 57

$\begin{array}{ll}\text { Bibliography } & 60\end{array}$

Vita

69 


\title{
STOCHASTIC FORMS OF FUNCTIONAL \\ ISOPERIMETRIC INEQUALITIES \\ Jesus Rebollo Bueno \\ Dr. Peter Pivovarov, Dissertation Supervisor
}

\begin{abstract}
The Brunn-Minkowski and Prékopa-Leindler inequalities admit a variety of proofs that are inspired by convexity. Nevertheless, the former holds for compact sets and the latter for integrable functions, so it seems that convexity has no special significance. On the other hand, it was recently shown that the BrunnMinkowski inequality, specialized to convex sets, follows from a local stochastic dominance for naturally associated random polytopes. In addition, a number of other geometric inequalities for convex sets arising from Brunn's concavity principle have recently been shown to yield local stochastic formulations, e.g., the Blaschke-Santaló inequality.

In the first part of this dissertation, we study reverse inequalities for functionals of polar convex bodies invariant under the general linear group. We strengthen planar isoperimetric inequalities; we do that by attaching a stochastic model to some classical ones, such as Mahler's Theorem, and a reverse Lutwak-Zhang inequality, for the polar of $L_{p}$ centroid bodies. In particular, we obtain the dual counterpart to a result of Bisztriczky-Böröczky.

For the rest of the dissertation, we initiate a systematic study of stochastic isoperimetric inequalities for random functions. We show that for the subclass of log-concave functions and associated stochastic approximations, a similar stochastic dominance underlies the Prékopa-Leindler inequality. Ultimately, we extend the latter result by developing stochastic geometry of $s$-concave functions. In this way, we establish local versions of dimensional forms of Brunn's principle à la Borell, Brascamp-Lieb, and Rinott. To do so, we define shadow systems of $s$ concave functions and revisit Rinott's approach in the context of multiple integral rearrangement inequalities.
\end{abstract}




\section{Chapter 1}

\section{Introduction}

Isoperimetric inequalities in convex geometry relate geometric (shape) and physical (measure) quantities of two objects. A first example is the classical isoperimetric inequality, after which all the other ones are named, which states that of all curves with the same perimeter, the circle encloses the largest area. This is just one example within Brunn-Minkowski theory, a rich framework which can be used in studying concepts like volume, surface area, and mean width under the effect of Minkowski addition of convex sets. In what follows, we discuss the content of this dissertation.

The essence of this work is that - in the presence of convexity - functional extensions of isoperimetric principles admit stronger stochastic versions. Inspired by work in stochastic geometry, we study operations between functions, such as the sup-convolution, inf-convolution, and approximations like the least concave majorant. As it happens, these are related to key operations in classical Brunn-Minkowski theory: Minkowski sums, convex hulls, $L_{p}$-sums, polar duals, etc. Given a convex function $f$, we generate a random model $[f]_{N}$ using, e.g., the least concave majorant of $N$ random points, or by revolving Euclidean balls with random centers. Inequalities for functionals $\varphi$ e.g., the integral of $f$, then hold locally, i.e., for the distribution function of the random variable $\varphi\left([f]_{N}\right)$. Fundamental inequalities can be treated in this framework, including the Prékopa-Leindler 
inequality and its dimension-dependent extension. These inequalities then arise as limiting cases via laws of large numbers.

\subsection{Sylvester's dual and planar inequalities}

We begin the work in this dissertation by studying functionals of convex bodies invariant under the general linear group, $G L(n)$. To pave the way, we begin with a fundamental example. Let $K$ be a convex body in $\mathbb{R}^{n}$, i.e., a compact convex set with non-empty interior. Whenever $K$ is symmetric with respect to the origin the volume product is defined by

$$
P(K):=|K|\left|K^{\circ}\right|
$$

where $|\cdot|$ denotes the $n$-dimensional Lebesgue measure and $K^{\circ}$ is the polar body of $K$ (see $\S 2.1$ for precise definitions). Since $P$ is continuous and $G L(n)$ invariant, a compactness argument shows that it attains a maximum and a minimum. The upper estimate is known as the Blaschke-Santaló inequality [10, 62, 85]:

$$
P(K) \leq P\left(B_{2}^{n}\right)
$$

where $B_{2}^{n}$ denotes the $n$-dimensional Euclidean unit ball centered at the origin. In addition, Alexander, Fradelizi, and Zvavitch [1] recently showed that among polytopes simplicial ones are maximizers; see [64] for earlier work in the plane. On the other hand, the lower estimate is known as the Mahler's Conjecture, confirmed by Mahler for dimension 2 [58], and it is still one of the main open problems in convex geometry:

$$
P(K) \geq P(L)
$$

where $L$ denotes the $n$-parallelotope for the symmetric case, or a simplex for the non-symmetric one. A recent breakthrough by Iriyeh and Shibata [43] solves it in dimension 3 (see Fradelizi, Hubard, Meyer, Roldán-Pensado, and Zvavitch [31] for 
a shorter proof). The $n$-dimensional statement is known in special cases. Saint Raymond established it for unconditional bodies [84] (see Meyer [61] for a simpler proof). Reisner proved the result for zonoids [78]. For local versions and other known special cases see $[7,44,45,53,67,79,91]$. Moreover, the celebrated result by Bourgain and Milman [13] established (1.3) up to a constant. See [38, 66, 73] for other proofs and related results. The sharpest known constant is due to Kuperberg [49].

On the topic of functional inequalities related to convex bodies, Paouris and Pivovarov studied stochastic forms of isoperimetric inequalities [68]. Subsequently, Cordero-Erausquin, Fradelizi, Paouris, and Pivovarov [26] developed randomized inequalities for polar bodies associated to (1.2). A typical example of such random sets is given by the convex hull of the columns of a random matrix, for which the expectation of the volume is maximized by $N$ independent random vectors uniformly distributed in the Euclidean ball of volume one. In particular, motivated by work in Stochastic Geometry [9, 18, 21, 22, 23, 41], they derived a stochastic Blashke-Santaló inequality, which we now recall. Let $K$ be a symmetric convex body in $\mathbb{R}^{n},\left\{X_{i}\right\}_{i=1}^{N}$ sampled uniformly on $K$, and $\left\{Y_{i}\right\}_{i=1}^{N}$ sampled uniformly on $K^{*}$, the Euclidean ball of the same volume as $K$. Then

$$
\mathbb{E}\left|\left([K]_{N}\right)^{\circ}\right| \leq \mathbb{E}\left|\left(\left[K^{*}\right]_{N}\right)^{\circ}\right|
$$

where $[K]_{N}$ stands for $\operatorname{conv}\left\{ \pm X_{1}, \ldots, \pm X_{N}\right\}$ and similarly for $\left[K^{*}\right]_{N}$. This example is indicative of recent research on isoperimetric inequalities: when an isoperimetric principle for convex sets can be proved by symmetrization, it is fruitful to instead carry out the symmetrization on product probability spaces. Multiple integral rearrangement inequalities of Rogers [82], Brascamp-Lieb-Luttinger [16], and Christ [24] then enter the picture and yield stronger stochastic formulations. This builds on principles in stochastic geometry e.g., [18, 21, 41]. One may find the origin of this in 1864, when J.J. Sylvester [92] posed his four-point problem, 
which ultimately led to the study of

$$
M(K)=\frac{1}{|K|} \mathbb{E}\left|[K]_{N}\right|
$$

for $N \geq n+1$. Thus, Sylvester's problem is equivalent to study the extremals of $M(K)$. Blaschke [9] showed that in two dimensions the minimum is attained if and only if $K$ is an ellipse, and triangles are the only maximizers of 1.4 for $N=4$ (see [30] for the extension to $N$ points on the plane). Indeed, since $M(K)$ is nonincreasing under Steiner symmetrization [40], ellipsoids are the only minimizers for the $n$-dimensional Sylvester's problem. The maximum problem is still open for $n>3$ and, as mentioned before, it is conjectured that the simplices are the only maximizers. Moreover, Campi, Colesanti, and Gronchi [21] used Rogers-ShephardMovements (see §2.2) to determine maximizers of the $r$-th order moment of (1.4) for $N>n=2$. See Meckes [59] for the symmetric case and for related uniqueness questions see Saroglu [86]. For the most recent developments linking sharp upper bounds in higher dimensions to Mahler's theorem, see work of Klartag [47]; see $[26,68,69,77]$ for further background.

We are interested in the polar version of Sylvester's functional. Let $K$ be a centrally symmetric convex body in $\mathbb{R}^{n},\left\{X_{i}\right\}_{i=1}^{n}$ independent random vectors sampled uniformly in $K$, and $r>0$. We study a generalization of the functional

$$
W(K)=\frac{1}{|K|} \mathbb{E}\left|\left([K]_{N}^{r}\right)^{\circ}\right|^{-1}
$$

where $N>n$, and $[K]_{N}^{r}=[K]_{N}+r B_{2}^{n}$. Notice, due to the negative moments, we need to add the $n$-dimensional Euclidean ball of radius $r, r B_{2}^{n}$, in order to ensure the integrability of the expression. In particular, we are interested in the functional of the normalized higher order moments of the volume of the polar of a random polytope in a centrally symmetric convex body in $\mathbb{R}^{n}$. Such functional, as Sylvester's, is continuous with respect to the Hausdorff metric and invariant under invertible linear transformations. 
The convexity of the function $t \mapsto W\left(K_{t}\right)$, for $K_{t}$ being a Rogers-ShephardMovement of $K$, Theorem 2.2, together with the setting beyond convex hull from Paouris and Pivovarov [68], provides a path to prove stochastic inequalities on the plane that strengthen planar isoperimetric inequalities. In particular, for centrally symmetric convex bodies $K$ in $\mathbb{R}^{2}$ with the origin as an interior point and $N \geq 2$, we are able to find a stochastic Mahler's inequality.

Theorem 1.1. Let $N \geq 2, r>0$, and $K$ a centrally symmetric convex body in $\mathbb{R}^{2}$ with the origin in its interior. Then

$$
\mathbb{E}\left|\left([K]_{N}^{r}\right)^{\circ}\right|^{-1} \leq \mathbb{E}\left|\left([Q]_{N}^{r}\right)^{\circ}\right|^{-1}
$$

where $Q$ is a centered square with $|K|=|Q|$.

In particular, by the Law of Large Numbers, when $N \rightarrow \infty$ and $r \rightarrow 0$ the latter random sets converge to their ambient bodies so (1.5) implies

$$
\left|K^{\circ}\right|^{-1} \leq\left|Q^{\circ}\right|^{-1} \text {, with }|K|=|Q|
$$

Thus, this shows Mahler's theorem holds "locally" for naturally associated random polytopes, not just the bodies $K$ and $L$. This is one example of a stochastic form of an isoperimetric inequality where the extremizers are not balls, ellipsoids, or rotationally invariant. Similarly, we will also be able to derive a stochastic reverse Lutwak-Zhang's inequality on the plane. Let $K$ be a star body about the origin in $\mathbb{R}^{n}$ and $1 \leq p \leq \infty$. The $p$-centroid body of $K, Z_{p} K$, is defined to be the convex body given by the function

$$
h\left(Z_{p} K, x\right)=\left(\frac{1}{|K|} \int_{K}|\langle x, z\rangle|^{p} d z\right)^{1 / p} .
$$

In [56] it is shown that

$$
\left|\left(Z_{p} K\right)^{\circ}\right| \leq\left|\left(Z_{p} B_{2}^{n}\right)^{\circ}\right| \text {, when }|K|=\left|B_{2}^{n}\right| \text {. }
$$


Thus, one can think of the latter as a generalization of the Blaschke-Santaló inequality (1.2), since it can be obtained as the limit case $p=\infty$ for (1.7). For more recent developments see $[54,55]$. A sharp reverse inequality on the plane was shown by Campi and Gronchi [22]

$$
\left|\left(Z_{p} K\right)^{\circ}\right| \geq\left|\left(Z_{p} Q\right)^{\circ}\right|, \text { when }|K|=|Q| \text {. }
$$

In order to give a stochastic form of (1.8) on the plane, let $K$ be a centrally symmetric convex body in $\mathbb{R}^{2}, N \geq 2,\left\{X_{i}\right\}_{i=1}^{N}$ independent random vectors uniformly distributed in $K, r>0$, and $1 \leq p \leq \infty$. In [26, 68, 69] stochastic strengthenings of isoperimetric inequalities for centroid bodies are established. There, the empirical $p$-centroid body of $K, Z_{p, N}(K)$, is defined by its (random) support function

$$
h^{p}\left(Z_{p, N}(K), z\right)=\frac{1}{N} \sum_{i=1}^{N}\left|\left\langle X_{i}, z\right\rangle\right|^{p} .
$$

Then, for example, stochastic dominance underlies (1.7)

$$
\mathbb{P}\left(\left|\left(Z_{p, N}(K)\right)^{\circ}\right|>t\right) \leq \mathbb{P}\left(\left|\left(Z_{p, N}\left(B_{2}^{n}\right)\right)^{\circ}\right|>t\right), \text { for } t>0
$$

We complement the latter with a reverse inequality. We denote $Z_{p, N, r}^{\circ}(K)=$ $\left(Z_{p, N}(K)+r B_{2}^{n}\right)^{\circ}$, and $Z_{N} K$ the empirical centroid body of $K$, for the case $p=1$.

Theorem 1.2. Let $K$ be a centrally symmetric convex body in $\mathbb{R}^{2}$ with the origin in its interior. Then

$$
\mathbb{E}\left|Z_{p, N, r}^{\circ}(K)\right|^{-1} \leq \mathbb{E}\left|Z_{p, N, r}^{\circ}(Q)\right|^{-1}
$$

where $|K|=|Q|$. In particular, when $N \rightarrow \infty$

$$
\left|\left(Z_{p} K\right)^{\circ}\right| \geq\left|\left(Z_{p} Q\right)^{\circ}\right|
$$

Bisztriczky and Böröczky [8] provided a planar converse of the Busemann- 
Petty centroid inequality $[18,19,70,71]$. We recall the latter states that given an origin symmetric convex body $K \subset \mathbb{R}^{n}$ then

$$
|Z K| \geq\left|Z B_{2}^{n}\right|, \text { when }|K|=\left|B_{2}^{n}\right|
$$

where $Z K$ denotes the centroid body, i.e., the case $p=1$. Bisztriczky and Böröczky showed that given a centrally symmetric convex body $K \subset \mathbb{R}^{2}$

$$
|Z K| \leq|Z Q|, \text { when }|K|=|Q|
$$

The following theorem gives a stochastic polar version of such inequality.

Theorem 1.3. Let $K$ be a centrally symmetric convex body in $\mathbb{R}^{2}$ with the origin as an interior point, and $|K|=|Q|$. Then

$$
\mathbb{E}\left|Z_{N, r}^{\circ}(K)\right|^{-1} \leq \mathbb{E}\left|Z_{N, r}^{\circ}(Q)\right|^{-1}
$$

In particular, when $N \rightarrow \infty$ and $r \rightarrow 0$

$$
\left|(Z K)^{\circ}\right| \geq\left|(Z Q)^{\circ}\right|
$$

Furthermore, we will be able to generalize Theorem 2.2 for non-symmetric convex bodies $K$ in $\mathbb{R}^{n}$ considering a generalization of the functional

$$
W^{s \circ}(K)=\frac{1}{|K|} \mathbb{E}\left|\left([K]_{N}^{r}\right)^{s \circ}\right|^{-1}
$$

where $N \geq n+1,[K]_{N}^{r}=\operatorname{conv}\left\{X_{1}, \ldots, X_{N}\right\}+r B_{2}^{n}$, and $K^{s \circ}$ denotes the polar body of $K$ with respect to its Santaló point. This will allow us to prove a stochastic non-symmetric Mahler's inequality.

Theorem 1.4. Let $K$ be a convex body in $\mathbb{R}^{2}$ with the origin in its interior, $r>0$, and $N \geq 3$. Then

$$
\mathbb{E}\left|\left([K]_{N}^{r}\right)^{s \circ}\right|^{-1} \leq \mathbb{E}\left|\left([T]_{N}^{r}\right)^{s \circ}\right|^{-1},
$$


where $T$ denotes a triangle with centroid at the origin and $|K|=|T|$.

We make special use of the work by Rogers and Shephard [81, 89] and developed by Campi, Colesanti, and Gronchi [21, 22, 23]. For more about shadow systems see Saroglou [87].

\subsection{Stochastic Prékopa-Leinder via bodies of rev- olution}

The Brun-Minkowski inequality is the cornerstone of Brunn-Minkowski theory. It governs the behavior of volume and Minkowski addition of compacts sets $K, L \subseteq$ $\mathbb{R}^{n}$ :

$$
|K+L|^{1 / n} \geq|K|^{1 / n}+|L|^{1 / n}
$$

see $[35,88]$. As an isoperimetric principle, (1.15) can equivalently be stated in the form

$$
|K+L| \geq\left|K^{*}+L^{*}\right|
$$

where $A^{*}$ denotes the Euclidean ball of the same volume as $A$. In this way, the Brunn-Minkowski inequality implies the standard isoperimetric theorem. In [69], it is shown that when one specializes to convex bodies $K, L \subseteq \mathbb{R}^{n}$, then (1.16) admits a stronger stochastic formulation for random polytopes. Namely, for each convex body $K \subseteq \mathbb{R}^{n}$, we sample independent random vectors $X_{1}, \ldots, X_{N}$ uniformly in $K$ and associate a random polytope

$$
[K]_{N}=\operatorname{conv}\left\{X_{1}, \ldots, X_{N}\right\}
$$

where conv means convex hull. Then sampling independent random vectors in each of the bodies in $(1.16)$ (on a common underlying probability space $(\Omega, \mathcal{A}, \mathbb{P})$ ) 
leads to the following for each $\alpha \geq 0$,

$$
\mathbb{P}\left(\left|[K]_{N}+[L]_{M}\right|>\alpha\right) \geq \mathbb{P}\left(\left|\left[K^{*}\right]_{N}+\left[L^{*}\right]_{M}\right|>\alpha\right) .
$$

See Figure 1.1.By the law of large numbers, as $N, M \rightarrow \infty$, the random polytopes converge to their ambient bodies.

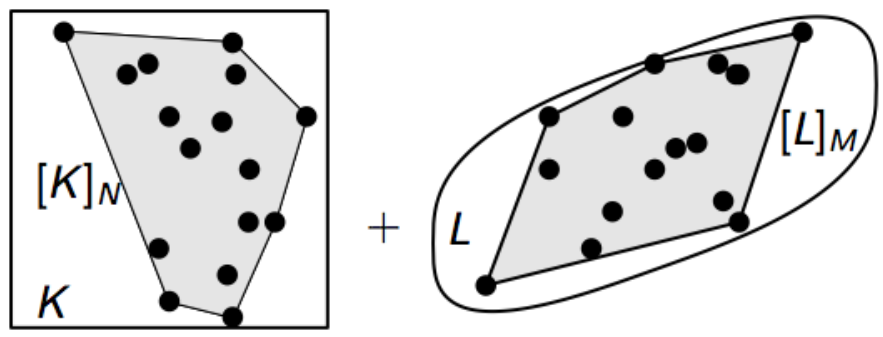

Figure 1.1: Stochastic approximation of convex bodies $K$ and $L$ from within by random polytopes $[K]_{N}$ and $[L]_{M}$, respectively

Thus(1.16) follows from a "local" stochastic dominance for random polytopes that naturally approximate convex bodies. Inequalities for the expected volume of the polytopes $[K]_{N}$ have a long history in stochastic geometry, including the previously mentioned Blaschke's resolution of Sylvester's four point problem [9], and its generalizations to higher dimensions by Busemann [18] and Groemer [41]. We explicitly mention only Groemer's inequality on random polytopes which is recovered from (1.17) when working with one body $K$ :

$$
\mathbb{E}\left|[K]_{N}\right| \geq \mathbb{E}\left|\left[K^{*}\right]_{N}\right|
$$

For background and related work, see, e.g., [21, 39, 68, 69], [88, Chapter 10] and the references therein.

The Prékopa-Leindler inequality is a natural extension of the Brunn-Minkowski inequality from sets to functions; it similarly provides a foundation for fundamental inequalities in analysis. The inequality $[51,75,76]$ asserts that for integrable 
functions $f, g, h: \mathbb{R}^{n} \rightarrow[0, \infty)$ and $0<\lambda<1$, if

$$
h(\lambda x+(1-\lambda) y) \geq f(x)^{\lambda} g(y)^{1-\lambda}
$$

for all $x, y \in \mathbb{R}^{n}$, then

$$
\int h \geq\left(\int f\right)^{\lambda}\left(\int g\right)^{1-\lambda}
$$

By taking $f=\mathbb{1}_{K}, g=\mathbb{1}_{L}$, and $h=\mathbb{1}_{\lambda K+(1-\lambda) L}$ (1.20) implies (1.15). In this sense the Prékopa-Leindler inequality can be viewed as a functional extension of the Brunn-Minkowski inequality. Related variations of (1.20) provide a basis for functional versions of Brunn's principle; see Borell [12], Rinott [80], Brascamp and Lieb $[14,15]$. The reach of the Prékopa-Leindler inequality now extends into various branches of analysis, geometry, probability, information theory, among other fields; see, e.g., [25, 27, 28, 29, 35, 57] and the references therein.

\subsection{Stochastic Brunn's principle via epigraphs and hypographs}

As for the Brunn-Minkowski inequality, our focus in Chapter 3 is on a form of (1.20) involving symmetric decreasing rearrangements $f^{*}$ and $g^{*}$ (see $§ 3.1$ for definitions). Brascamp and Lieb proved (1.20) via rearrangement inequalities through the reverse Young inequality [14]. More recently, Melbourne [60] derived families of rearrangement inequalities refining (1.20); the version we study here involves Borel measurable $f, g: \mathbb{R}^{n} \rightarrow[0, \infty), 0<\lambda<1$ and the sup-convolution

$$
\left(f \star_{\lambda} g\right)(v)=\sup \left\{f^{\lambda}(x) g^{1-\lambda}(y): v=\lambda x+(1-\lambda) y\right\}
$$

for which one has

$$
\int_{\mathbb{R}^{n}}\left(f \star_{\lambda} g\right)(v) d v \geq \int_{\mathbb{R}^{n}}\left(f^{*} \star_{\lambda} g^{*}\right)(v) d v .
$$


We will show that a similar "local" stochastic dominance underlies (1.21) when one focuses only on $\log$-concave functions $f$ and $g$, i.e., when $\log f$ and $\log g$ are concave on their supports.

To define our stochastic model, for each integrable log-concave function $f$ : $\mathbb{R}^{n} \rightarrow[0, \infty)$, we sample independent random vectors $\left(X_{1}, Z_{1}\right), \ldots,\left(X_{N}, Z_{N}\right)$ in $\mathbb{R}^{n} \times[0, \infty)$ according to the uniform Lebesgue measure on the region under the graph of $f$ :

$$
G_{f}:=\left\{(x, z) \in \mathbb{R}^{n} \times[0, \infty): x \in \operatorname{supp} f, z \leq f(x)\right\}
$$

We denote by $[f]_{N}$ the least log-concave function supported on $\operatorname{conv}\left\{X_{1}, \ldots, X_{N}\right\}$ with $[f]_{N}\left(X_{i}\right) \geq Z_{i}, i=1, \ldots, N$. In other words, for the convex domain

$$
H_{f, N}:=\operatorname{conv}\left\{\left(X_{1}, \log Z_{1}\right), \ldots,\left(X_{N}, \log Z_{N}\right)\right\}
$$

we set

$$
[f]_{N}(x):=\exp \left(\sup \left\{z:(x, z) \in H_{f, N}\right\}\right)
$$

This model of approximation works equally well for $f^{*}$. Indeed, when $f$ is $\log$ concave, the same is true of $f^{*}$. Thus for independent random samples for each of the log-concave functions $f, g, f^{*}, g^{*}$, we can now state our main result.

Theorem 1.5. Let $f, g: \mathbb{R}^{n} \rightarrow[0, \infty)$ be log-concave functions and $N, M>n+1$. Then, for any $\alpha>0$,

$$
\mathbb{P}\left(\int_{\mathbb{R}^{n}}\left([f]_{N} \star_{\lambda}[g]_{M}\right)(v) d v>\alpha\right) \geq \mathbb{P}\left(\int_{\mathbb{R}^{n}}\left(\left[f^{*}\right]_{N} \star_{\lambda}\left[g^{*}\right]_{M}\right)(v) d v>\alpha\right)
$$

When $N, M \rightarrow \infty$, the latter implies (1.21). Thus, in this sense, a "local" stochastic dominance underlies the Prékopa-Leindler inequality for log-concave $f$ and $g$.

There is a solid foundation for extending geometric inequalities from convex sets to log-concave functions or more general classes, e.g., $[2,4,6,11,32,48$, 
$50,65]$. While a number of isoperimetric inequalities in addition to (1.15) have found stochastic versions [69], no similar progress has been made for stochastic functional inequalities. Theorem 1.5 is a first step towards stochastic isoperimetric inequalities for random functions. Working with just one function $f$, we obtain a functional analogue of Groemer's result (1.18) for random polytopes.

Theorem 1.6. Let $f: \mathbb{R}^{n} \rightarrow[0, \infty)$ be an integrable log-concave function and $N>n+1$. Then, for any $\alpha>0$,

$$
\mathbb{P}\left(\int_{\mathbb{R}^{n}}[f]_{N}(x) d x>\alpha\right) \geq \mathbb{P}\left(\int_{\mathbb{R}^{n}}\left[f^{*}\right]_{N}(x) d x>\alpha\right)
$$

The techniques from [69] leading to the stochastic Brunn-Minkowski inequality (1.17) involve random convex sets. New ingredients are needed to treat functions and their stochastic approximations. Various proofs of the Prékopa-Leindler inequality (1.20) rely on the Brunn-Minkowski inequality. Of particular significance to our approach is the work of Klartag [46]; he derives various functional inequalities, including (1.20), as "marginals" of geometric inequalities for convex bodies in higher dimensions. The reduction to sets in higher dimensions fits well with the stochastic approximation that we use. In particular, they interface well with multiple integral rearrangement inequalities of Rogers [82], Brascamp-Lieb-Luttinger [16] and Christ [24].

Lastly, Brunn's concavity principle underpins a wealth of inequalities in geometry and analysis. One can formulate it as follows: for any convex body $K \subseteq \mathbb{R}^{n}$ and any direction $\theta$, the $(n-1)$-volume of slices of $K$ by parallel translates of $\theta^{\perp}$ is $1 /(n-1)$-concave on its support, i.e.,

$$
A(t)=\left|K \cap\left(\theta^{\perp}+t \theta\right)\right|^{1 /(n-1)}
$$

is concave. A far-reaching extension of this principle in analysis is exemplified by a family of functional inequalities obtained by Borell [12] and Brascamp-Lieb [15], with an alternate approach put forth by Rinott [80]. These inequalities can 
be formulated in terms of certain means as follows: for $a, b \geq 0, s \geq-1 / n$ and $\lambda \in(0,1)$, set

$$
\mathcal{M}_{\lambda}^{s}(a, b)= \begin{cases}\left(\lambda a^{s}+(1-\lambda) b^{s}\right)^{1 / s} & \text { if } a b \neq 0 \\ 0 & \text { if } a b=0\end{cases}
$$

where the cases $s \in\{-1 / n, 0,+\infty\}$ are defined as limits

$$
\mathcal{M}_{\lambda}^{-1 / n}(a, b)=\min \{a, b\}, \quad \mathcal{M}_{\lambda}^{+\infty}(a, b)=\max \{a, b\}, \quad \mathcal{M}_{\lambda}^{0}(a, b)=a^{\lambda} b^{1-\lambda} .
$$

Then for measurable functions $f, g, h: \mathbb{R}^{n} \rightarrow[0, \infty), 0<\lambda<1$, and $s \geq-1 / n$, if

$$
h\left(\lambda x_{1}+(1-\lambda) x_{2}\right) \geq \mathcal{M}_{\lambda}^{s}\left(f\left(x_{1}\right), g\left(x_{2}\right)\right)
$$

for all $x_{1}, x_{2} \in \mathbb{R}^{n}$, one has

$$
\int_{\mathbb{R}^{n}} h \geq \mathcal{M}_{\lambda}^{s /(1+n s)}\left(\int_{\mathbb{R}^{n}} f, \int_{\mathbb{R}^{n}} g\right)
$$

The Prékopa-Leindler inequality (1.20) corresponds to the logarithmically concave case $s=0$; for earlier work on the real line, see Henstock and Macbeath [42]. These principles are now fundamental in analysis, geometry and probability, among other fields. For their considerable impact, we refer the reader to [35] and the references therein.

The inequalities (1.27) stem from principles rooted in convexity. Indeed, the standard approach to Brunn's principle (1.25) connects concavity of the map $t \mapsto A(t)$ to the convexity of $K$ through suitable symmetrizations, see e.g., [3]. In [14], Brascamp and Lieb used symmetrization to prove certain cases of (1.27). Subsequently, they provided an alternate inductive approach, based on the Brunn-Minkowski inequality [15]. Rinott provided an alternate proof, starting with epigraphs of convex functions [80]. However, the inequalities ultimately do not require convexity as they hold for measurable functions. So convexity (or 
concavity) of the functions involved seems of no importance. In Chapter 4, our focus is on what more can be said when the functions involved do possess some concavity.

The work initiated on the Prékopa-Leindler inequality for random log-concave functions, is then extended to the full family of inequalities (1.27). We prove they actually have "local" stochastic strengthenings for functions $f$ that are $s$-concave, i.e. $f^{s}$ is concave on its support; when $s<0$, this means that $f^{s}$ is convex. To formulate our main result, for each $s$-concave function $f$, we sample independent random vectors $\left(X_{1}, Z_{1}\right), \ldots,\left(X_{N}, Z_{N}\right)$ distributed uniformly under the graph of $f$ according to Lebesgue measure. We associate random functions $[f]_{N}$, supported on the convex hull conv $\left\{X_{1}, \ldots, X_{N}\right\}$, defined by

$$
[f]_{N}(x)= \begin{cases}\inf \left\{z^{1 / s}:(x, z) \in \mathcal{P}_{f, N}\right\}, & \text { if } s<0 \\ \sup \left\{e^{z}:(x, z) \in \mathcal{P}_{f, N}\right\}, & \text { if } s=0, \\ \sup \left\{z^{1 / s}:(x, z) \in \mathcal{P}_{f, N}\right\}, & \text { if } s>0,\end{cases}
$$

where

$$
\mathcal{P}_{f, N}= \begin{cases}\operatorname{conv}\left\{\left(X_{1}, Z_{1}^{s}\right), \ldots,\left(X_{N}, Z_{N}^{s}\right)\right\}, & \text { if } s \neq 0 . \\ \operatorname{conv}\left\{\left(X_{1}, \log Z_{1}\right), \ldots,\left(X_{N}, \log Z_{N}\right)\right\}, & \text { if } s=0 .\end{cases}
$$

In other words, when $s=0$ or $s>0,[f]_{N}$ is the least log-concave or $s$-concave function, respectively, satisfying $[f]_{N}\left(X_{i}\right) \geq Z_{i}$; similarly, when $s<0,[f]_{N}$ is the greatest $s$-concave function with $[f]_{N}\left(X_{i}\right) \leq Z_{i}$. See Figure 1.2 for the case $s=0$.

With this notation, we can state our main result, which we formulate in terms of the sup-convolution

$$
\left(f \star_{\lambda, s} g\right)(v)=\sup \left\{\mathcal{M}_{\lambda}^{s}\left(f\left(x_{1}\right), g\left(x_{2}\right)\right): v=\lambda x_{1}+(1-\lambda) x_{2}\right\}
$$

and the symmetric decreasing rearrangements $f^{*}$ and $g^{*}$ of $f$ and $g$, respectively 


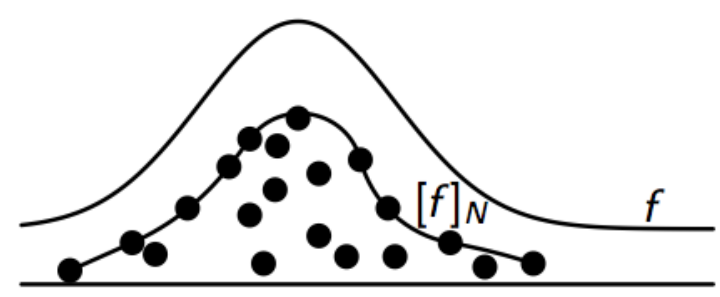

Figure 1.2: Stochastic approximation of a log-concave function $f$ by using the least log-concave majorant $[f]_{N}$ above a random sample under the graph of $f$.

(defined in (3.1)).

Theorem 1.7. Let $s \in(-1 / n, \infty)$ and let $f, g: \mathbb{R}^{n} \rightarrow[0, \infty)$ be integrable $s$ concave functions and $N, M>n+1$. Then for $\alpha>0$,

$$
\mathbb{P}\left(\int_{\mathbb{R}^{n}}\left([f]_{N} \star_{\lambda, s}[g]_{M}\right)(v) d v>\alpha\right) \geq \mathbb{P}\left(\int_{\mathbb{R}^{n}}\left(\left[f^{*}\right]_{N} \star_{\lambda, s}\left[g^{*}\right]_{M}\right)(v) d v>\alpha\right) .
$$

When $N, M \rightarrow \infty$ one gets (1.21). As mentioned, Brascamp and Lieb's first approach to cases of (1.27) used rearrangements. Recent interest in rearranged strengthenings for this and other means in (1.27) have been studied by Melbourne [60] for general functions. Roysdon and Xing have studied $L_{p}$ variants of the Borell-Brascamp-Lieb inequality [83].

Special cases of Theorem 1.7, namely $s=1 / q(q \in \mathbb{N})$ were treated in [74]. The approach used multiple integral rearrangement inequalities (as discussed above) and, additionally, built on ideas of Artstein-Avidan, Klartag and Milman [4] on moving from convex sets to log-concave functions. Here a new key step is inspired by Rinott's approach to (1.27) via epigraphs of convex functions [80]; the latter has recently been used in a dual setting by Artstein-Avidan, Florentin and Segal [2] for a new Prékopa-Leindler inequality.

Another new tool developed in this chapter is that of linear parameter systems for $s$-concave functions. Linear parameter systems along a direction $\theta \in \mathbb{S}^{n-1}$ are 
families of convex sets of the form

$$
K_{t}=\operatorname{conv}\left\{x_{i}+\lambda_{i} t \theta: i \in I\right\}, \quad t \in[a, b],
$$

where $I$ is an index set, $\left\{x_{i}\right\}_{i \in I} \subseteq \mathbb{R}^{n}$ and $\left\{\lambda_{i}\right\}_{i \in I} \subseteq \mathbb{R}$ are bounded sets. Rogers and Shephard [81] proved the fundamental fact that the volume of $K_{t}$ is a convex function of $t$. This was extended to the more general notion of shadow systems by Shephard [89]. Shadow systems encorporate key features of Steiner symmetrization and have been successfully used in a variety of isoperimetric type inequalities, developed especially by Campi and Gronchi, e.g., [22, 23]; for other examples, see $[34,63,87,93]$ or $[88]$ and the references therein.

The proof of Theorem 1.7 relies on extending linear parameter systems to $s$ concave functions. Let $I$ be an index set, and $\left\{\left(x_{i}+\lambda_{i} t \theta, z_{i}\right)\right\}_{i \in I} \subseteq \mathbb{R}^{n} \times[0, \infty)$ a collection of points lying under the graph of an integrable $s$-concave function and set $w_{i}(t)=x_{i}+\lambda_{i} t \theta, i \in I$. Analogous to the definition of $[f]_{N}$, we define, for $s \geq 0, f_{t, s}$ to be the least $s$-concave function above $\left\{w_{i}(t)\right\}$; similarly, for $s<0$, we define $f_{t, s}$ to be the greatest $s$-concave function beneath the points $\left\{w_{i}(t)\right\}$ (see $\S 4.3)$. In this setting, we show that

$$
t \mapsto \int_{\mathbb{R}^{n}} f_{t, s}(v) d v
$$

is convex. Just as linear parameter systems can be viewed as special shadow systems, the same applies to $s$-concave functions. We also give an interpretation of shadow systems of $s$-concave functions in terms of associated epigraphs and hypographs and establish related convexity properties in $\S 4.3$. We show in $\S 4.2$ how these interface with rearrangement inequalities and thus provide a path towards stochastic geometry of $s$-concave functions and associated extremal inequalities. 


\section{Chapter 2}

\section{Stochastic Reverse Isoperimetric Inequalities On The Plane}

Work in stochastic geometry has primarily concentrated on inequalities where the extremizer is a ball, an ellipsoid, or rotationally invariant. In this chapter, we study reverse inequalities where the extremizers are not balls, and associate stochastic forms to them. Since the extremals for reverse inequalities are typically unknown for arbitrary dimension, we work on the plane.

\subsection{Definitions and notation}

We denote by $B_{2}^{n}$ the Euclidean unit ball in $\mathbb{R}^{n}$ centered at the origin, i.e.,

$$
B_{2}^{n}:=\left\{x \in \mathbb{R}^{n}:\|x\|_{2} \leq 1\right\}
$$

Also $\mathbb{S}^{n-1}$ denotes the Euclidean unit sphere in $\mathbb{R}^{n}$ centered at the origin, that is,

$$
\mathbb{S}^{n-1}:=\left\{x \in \mathbb{R}^{n}:\|x\|_{2}=1\right\}
$$


A set is (centrally) symmetric if $K=-K$. The Minkowski sum of $K, L$ sets in $\mathbb{R}^{n}$ is given by

$$
K+L=\{k+l: k \in K, l \in L\}
$$

and the Hausdorff distance between $K$ and $L$ by

$$
\delta^{H}(K, L)=\inf \left\{\epsilon>0: K \subset L+\epsilon B_{2}^{n}, L \subset K+\epsilon B_{2}^{n}\right\}
$$

We call a set convex if for all $\lambda \in(0,1)$ and $x, y \in \mathbb{R}^{n}$, then $\lambda x+(1-\lambda) y \in K$. Let $K$ be a convex set, $\theta \in \mathbb{S}^{n-1}$, and $P_{\theta^{\perp}}$ the orthogonal projection onto $\theta^{\perp}$. We define the upper function of $K$ with respect to $\theta$ as

$$
\begin{aligned}
u_{K, \theta}: P_{\theta^{\perp}} K & \longmapsto \mathbb{R} \\
u_{K, \theta}(y) & =\sup \{\lambda: y+\lambda \theta \in K\} .
\end{aligned}
$$

Analogously we define the lower function of $K$ with respect to $\theta$ as

$$
\begin{aligned}
\ell_{K, \theta}: P_{\theta^{\perp}} K & \longmapsto \mathbb{R} \\
\ell_{K, \theta}(y) & =\inf \{\lambda: y+\lambda \theta \in K\} .
\end{aligned}
$$

Whenever $K$ and $\theta$ are clear by the context we will just write $P, u$, and $\ell$. Also notice that, for $K$ convex, $u$ and $\ell$ are concave and convex, respectively.

Let $\langle\cdot, \cdot\rangle$ be the usual inner product, the support function of a convex set is defined as

$$
h_{K}(w)=\max _{x \in K}\langle w, x\rangle \quad\left(w \in \mathbb{R}^{n}\right)
$$

One can define the $p$-centroid body of $K$ by its support function as

$$
h_{Z_{p} K}(z)=\left(\frac{1}{|K|} \int_{K}|\langle x, z\rangle|^{p} d x\right)^{1 / p} .
$$

Thus, the volume of $Z_{p} K$ and $Z_{p} \Phi K$ is the same whenever $\Phi$ is a linear trans- 
formation with determinant one. Indeed, the centroid body itself is an affine invariant.

The polar set, $K^{\circ}$, of $K$ is given by

$$
K^{\circ}=\left\{\omega \in \mathbb{R}^{n}:\langle\omega, x\rangle \leq 1, \forall x \in K\right\}
$$

Notice that $K^{\circ}$ depends on the location of the origin and it follows from the definition that if $K$ contains the origin $\left(K^{\circ}\right)^{\circ}=K$. In addition, a convex set is said to be a convex body if it is also compact with non-empty interior. In such case, the volume of the polar body of $K$ can be determined using the support function by

$$
\left|K^{\circ}\right|=\frac{1}{n} \int_{\mathbb{S}^{n-1}} h_{K}^{-n}(x) d x
$$

where denotes $|\cdot|$ the Lebesgue measure on $\mathbb{R}^{n}$. For the case of a non-symmetric convex body we define

$$
K^{s \circ}=(K-s)^{\circ}=\left\{\omega \in \mathbb{R}^{n}:\langle\omega, x-s\rangle \leq 1, \forall x \in K\right\}
$$

where $s$ denotes the Santaló point of $K$, i.e. the unique point in the interior of $K$ such that

$$
\left|K^{s \circ}\right|=\min _{x \in \operatorname{int}(\mathrm{K})}\left\{\left|(K-x)^{\circ}\right|\right\}
$$

For $\left\{x_{1}, \ldots, x_{N}\right\} \subset \mathbb{R}^{n}$ we denote by $[\mathbf{x}]:=\left[x_{1} \cdots x_{N}\right]$ the linear operator from $\mathbb{R}^{N}$ to $\mathbb{R}^{n}$, and $[\mathbf{x}] C$ the set

$$
[\mathbf{x}] C=\left\{\sum_{i=1}^{N} c_{i} x_{i}: c=\left(c_{i}\right) \in C\right\} \subset \mathbb{R}^{n} .
$$

An example of this is when we consider $C=B_{1}^{N}$, then

$$
[\mathbf{x}] B_{1}^{N}=\operatorname{conv}\left\{ \pm x_{1}, \ldots, \pm x_{N}\right\}
$$

where conv stands for convex hull. 


\subsection{Dual version of Sylvester's functional}

We begin by recalling the notion of shadow system introduced by Rogers and Shephard [81].

Definition 2.1. A shadow system along a direction $\theta \in \mathbb{S}^{n-1}$ is a family of convex sets $K_{t} \subset \mathbb{R}^{n}$ defined by

$$
K_{t}=\operatorname{conv}\left\{x+t \alpha(x) \theta: x \in A \subset \mathbb{R}^{n}\right\},
$$

where $A$ is an arbitrary bounded set of points, $\alpha$ is a bounded function on $A$, and $t$ belongs to an interval of the real axis.

Rogers and Shephard $[81,89]$ proved that the volume of $K_{t}$ is a convex function of $t$, and many isoperimetric type inequalities have been shown using this technique. In our case, the main feature of these movements we use is the following theorem due to Campi and Gronchi [22]:

Theorem 2.1. If $K_{t}, t \in[0,1]$, is a shadow system of origin symmetric convex bodies in $\mathbb{R}^{n}$, then $\left|K_{t}^{\circ}\right|^{-1}$ is a convex function of $t$.

We are interested in a particular case of shadow systems studied by Campi, Colesanti, and Gronchi [21], where the bounded function $\alpha$ is constant on each chord of $K$ parallel to $\theta$.

Definition 2.2. Let $K \subset \mathbb{R}^{n}$. A shadow system is called a Rogers-Shephardmovement of $K$ if

$$
K_{t}=\operatorname{conv}\{x+t \beta(P x) \theta: x \in K\},
$$

where $t \in[a, b], 0 \in[a, b], P$ is the orthogonal projection onto $\theta^{\perp}$, and $\beta$ is a real valued function on $P K$. 
Notice that, if $K_{t}$ is a Rogers-Shephard-movement then

$$
\begin{aligned}
K_{t}+r B_{2}^{n} & :=\operatorname{conv}\left\{x+r B_{2}^{n}+t \beta(P x) \theta: x \in K\right\} \\
& =\operatorname{conv}\left\{y+t \tilde{\beta}(P y) \theta: y \in K+r B_{2}^{n}\right\}
\end{aligned}
$$

for some $\tilde{\beta}$ with $\tilde{\beta}(y)=\beta(y)$ for $P y \in P K$.

Now, we introduce a functional motivated by Sylvester's. It expresses the normalized higher negative moments of the volume of the polar of a random polytope in $K$. Denote by $\mathcal{K}^{n}$ the class of all convex bodies in $\mathbb{R}^{n}$ and $\mathcal{K}_{0}^{n}$ the class of all convex bodies with the origin as an interior point. Let $K \in \mathcal{K}_{0}^{n}$ and $C \in \mathcal{K}_{0}^{N}$ be centrally symmetric, $p \geq 1, N \geq n, r>0$, and $X_{1}, \ldots, X_{N}$ independent random vectors sampled uniformly on $K$. We define the functional

$$
W_{p}^{r}(K ; N ; C)=\frac{1}{|K|^{p}} \mathbb{E}\left|\left(\left[X_{1} \cdots X_{N}\right] C+r B_{2}^{n}\right)^{\circ}\right|^{-p}
$$

Then, $W_{p}^{r}(K ; N ; C)$ is continuous with respect to the Hausdorff metric and it is invariant under invertible linear transformations.

Theorem 2.2. Let $C \in \mathcal{K}_{0}^{N}$ be centrally symmetric, $K_{t}$ a Rogers-ShephardMovement for $t \in[-1,1], p \geq 1$, and $N \geq n$. Then

$$
t \longmapsto W_{p}^{r}\left(K_{t} ; N ; C\right)
$$

is a convex function of $t$.

Proof. Suppose without loss of generality $K_{0}=K$, so $P K=P K_{t}$ and $|K|=\left|K_{t}\right|$ for all $t \in[-1,1]$. Let $u$ and $\ell$ be as in $\S 2.1$, then one has

$$
\begin{aligned}
K & =\{(x, y) \in P K \times \mathbb{R}: \ell(x) \leq y \leq u(x)\} \\
K_{t} & =\{(x, y) \in P K \times \mathbb{R}:(\ell+t \beta)(x) \leq y \leq(u+t \beta)(x)\} .
\end{aligned}
$$


Therefore by Fubini's Theorem

$$
\begin{aligned}
W_{p}^{r}\left(K_{t} ; N ; C\right) & =\frac{1}{|K|^{N+p}} \int_{(P K)^{N}}\left(\prod_{i=1}^{N} \int_{(\ell+t \beta)\left(x_{i}\right)}^{(u+t \beta)\left(x_{i}\right)}\left|\left(M_{1} C+r B_{2}^{n}\right)^{\circ}\right|^{-p} d \vec{y}\right) d \vec{x} \\
& =\frac{1}{|K|^{N+p}} \int_{(P K)^{N}}\left(\prod_{i=1}^{N} \int_{\ell\left(x_{i}\right)}^{u\left(x_{i}\right)}\left|\left(M_{2} C+r B_{2}^{n}\right)^{\circ}\right|^{-p} d \overrightarrow{\widetilde{y}}\right) d \vec{x},
\end{aligned}
$$

where

$$
M_{1}:=\left[x_{1}+y_{1} \theta \cdots x_{N}+y_{N} \theta\right]
$$

and after considering $y_{i}=\widetilde{y}_{i}+t \beta\left(x_{i}\right)$

$$
M_{2}:=\left[x_{1}+\left(\widetilde{y}_{1}+t \beta\left(x_{1}\right)\right) \theta \cdots x_{N}+\left(\widetilde{y}_{N}+t \beta\left(x_{N}\right)\right) \theta\right] .
$$

Notice (2.4) is convex since, by Theorem 2.1, it is the repeated integral of the $p$-th power of a convex functional.

At this point we generalize the previous functional for non-symmetric convex bodies. Let $N \geq n, K \in \mathcal{K}^{n}, C \in \mathcal{K}^{N}, X_{1}, \ldots, X_{N}$ independent random vectors sampled uniformly in $K, p \geq 1$, and $r>0$. The functional

$$
W_{p, r}^{s \circ}(K ; N ; C)=\frac{1}{|K|^{p}} \mathbb{E}\left|\left(\left[X_{1} \cdots X_{N}\right] C+r B_{2}^{n}\right)^{s \circ}\right|^{-p},
$$

expresses the normalized higher negative moments of the volume, of the polar of a non-symmetric random polytope in $K$ with respect to its Santaló point.

The following theorem by Meyer and Reisner [63], allows us to show for (2.6) an analogous result to Theorem 2.2. We recall a non-degenerate shadow system, $K_{t}$, is a shadow system with non-empty interior for all $t$ in the interval.

Theorem 2.3. Let $K_{t}, t \in[a, b]$, be a non-degenerated shadow system in $\mathbb{R}^{n}$. Then $\left|K_{t}^{s \circ}\right|^{-1}$ is a convex function of $t$. 
Notice (2.6) is continuous with respect to the Hausdorff metric, and invariant under invertible linear transformations. However, in general, the functional is not invariant under invertible affine transformations; unless considering $C=$ $\operatorname{conv}\left\{e_{1}, \ldots, e_{N}\right\}$. We focus on this case.

Theorem 2.4. Let $C=\operatorname{conv}\left\{e_{1}, \ldots, e_{N}\right\}, K_{t}$ a Rogers-Shephard-Movement for $t \in[-1,1], p \geq 1, r>0$, and $N \geq n+1$. Then

$$
t \longmapsto W_{p, r}^{s \circ}\left(K_{t} ; N ; C\right)
$$

is a convex function of $t$.

Proof. Suppose without loss of generality $K_{0}=K$, so $P K=P K_{t}$ and $|K|=\left|K_{t}\right|$ for all $t \in[-1,1]$. Let $u$ and $\ell$ be as in $\S 2.1$. Then, as in Theorem 2.2, the convexity of (2.6) follows by Fubini's Theorem and Theorem 2.3.

\subsection{Applications}

Here we present some applications of Theorem 2.2 and Theorem 2.4. In particular, we argue the maximizers for our functionals (2.4) and (2.6) are squares and triangles, respectively, and prove by a random approximation procedure important results from Convex Geometry in the plane where a local stochastic dominance holds. We start by showing the maximizers for (2.4).

Lemma 2.1. Let $N \geq 2, C \in \mathcal{K}_{0}^{N}$ origin symmetric, and $p \geq 1$. Then

$$
W_{p}^{r}(K ; N ; C) \leq W_{p}^{r}\left(B_{\infty}^{2} ; N ; C\right)
$$

for all centrally symmetric $K \in \mathcal{K}_{0}^{2}$.

Proof. Let $m \geq 3, K$ a polygon with $\left\{ \pm a_{1}, \ldots, \pm a_{m}\right\}$ ordered clockwise vertices, $\theta$ a direction parallel to the line joining $a_{1}$ and $a_{3}$, and set

$$
K_{t}:=\operatorname{conv}\left\{ \pm a_{1}, a_{2}+t \theta, \pm a_{3}, \ldots, \pm a_{m}\right\}
$$


Then, there exists $\delta_{1}, \delta_{2}>0$ such that for $t \in\left[-\delta_{1}, \delta_{2}\right], K_{t}$ is a Rogers-Shephardmovement where $K_{-\delta_{1}}$ and $K_{\delta_{2}}$ have $m-2$ vertices. As $W_{p}^{r}(K ; N ; C)$ is a convex functional, Theorem 2.2,

$$
W_{p}^{r}(K ; N ; C) \leq \max \left\{W_{p}^{r}\left(K_{-\delta_{1}} ; N ; C\right), W_{p}^{r}\left(K_{\delta_{2}} ; N ; C\right)\right\}
$$

and iterating the above procedure one has

$$
W_{p}^{r}(K ; N ; C) \leq W_{p}^{r}(P ; N ; C)
$$

where $P$ is a parallelogram. The result follows by the invariance under linear transformations and the continuity of the functional.

We recall now a convergence result that will allow us to recover the classical inequalities, for the proof we refer to [88].

Lemma 2.2. Let $K, K_{1}, \ldots, K_{N}$ be convex bodies with the origin as an interior point with $K_{N} \stackrel{\delta^{H}}{\longrightarrow} K$ as $N \rightarrow \infty$. Then

$$
K_{N}^{\circ} \stackrel{\delta^{H}}{\longrightarrow} K^{\circ} \text { as } N \rightarrow \infty .
$$

Let $(\Omega, \mathcal{F}, \mathbb{P})$ be a probability space, and assume we have the following independent random vectors sampled uniformly according to the Lebesgue measure:

- $\left\{X_{i}\right\}_{i=1}^{N}$ sampled in $K$.

- $\left\{Y_{i}\right\}_{i=1}^{N}$ sampled in $B_{\infty}^{2}$.

- $\left\{\widetilde{Y}_{i}\right\}_{i=1}^{N}$ sampled in a triangle $T$ of volume one and centroid the origin.

Denote $[\mathbf{X}],[\mathbf{Y}]$, and $[\tilde{\mathbf{Y}}]$ as in $\S 2.1$. We now recall that under almost sure convergence, e.g. [26], integration and limit operation can be interchanged, so by Lemma 2.2 one has the following $\mu$ almost surely convergence in $\delta^{H}$ : 
- $[\mathbf{X}] B_{1}^{N} \rightarrow K$

- $\frac{1}{N^{1 / p}}[\mathbf{X}] B_{q}^{N} \rightarrow Z_{p} K$.

- $\frac{1}{N}[\mathbf{X}] B_{\infty}^{N} \rightarrow Z K$
- $[\mathbf{Y}] B_{1}^{N} \rightarrow B_{1}^{2}$.

- $\frac{1}{N^{1 / p}}[\mathbf{Y}] B_{q}^{N} \rightarrow Z_{p} B_{\infty}^{2}$

- $\frac{1}{N}[\mathbf{Y}] B_{\infty}^{N} \rightarrow Z B_{\infty}^{2}$

Now, using Theorem 2.2 and Lemma 2.1, we deduce the proofs of the stochastic forms on the plane for Mahler's Theorem [58], Theorem 1.1, a reverse LutwakZhang's inequality [56], Theorem 1.2, and a polar Bisztriczky-Böröczky's inequality [8], Theorem 1.3.

Proof of Theorem 1.1. Consider without loss of generality $K$ such that $|K|=\left|B_{\infty}^{2}\right|$ and notice we are able to write

$$
[K]_{N}=[\mathbf{X}] B_{1}^{N} \quad \text { and } \quad\left[B_{\infty}^{2}\right]_{N}=[\mathbf{Y}] B_{1}^{N}
$$

By Lemma 2.1 with $C=B_{1}^{N}$ we have

$$
\frac{1}{|K|^{p}} \mathbb{E}\left|\left([\mathbf{X}] B_{1}^{N}+r B_{2}^{n}\right)^{\circ}\right|^{-p} \leq \frac{1}{\left|B_{\infty}^{2}\right|^{p}} \mathbb{E}\left|\left([\mathbf{Y}] B_{1}^{N}+r B_{2}^{n}\right)^{\circ}\right|^{-p}
$$

and inequality (1.5) follows.

Remark 2.1. From (1.5) one has

$$
\lim _{N \rightarrow \infty} \mathbb{E}\left|\left([\mathbf{X}] B_{1}^{N}+r B_{2}^{n}\right)^{\circ}\right|^{-1} \leq \lim _{N \rightarrow \infty} \mathbb{E}\left|\left([\mathbf{Y}] B_{1}^{N}+r B_{2}^{n}\right)^{\circ}\right|^{-1}
$$

Thus, by the continuity of the Lebesgue measure and Dominated Convergence Theorem

$$
\mathbb{E}\left|\left(\lim _{N \rightarrow \infty}[\mathbf{X}] B_{1}^{N}+r B_{2}^{n}\right)^{\circ}\right|^{-1} \leq \mathbb{E}\left|\left(\lim _{N \rightarrow \infty}[\mathbf{Y}] B_{1}^{N}+r B_{2}^{n}\right)^{\circ}\right|^{-1}
$$

Therefore by Lemma 2.2 one has the almost sure convergence mentioned above. It follows

$$
\left|\left(K+r B_{2}^{n}\right)^{\circ}\right| \geq\left|\left(B_{\infty}^{2}+r B_{2}^{n}\right)^{\circ}\right|
$$


for each $r>0$. Hence we can appeal to dominated convergence once more and let $r \rightarrow 0$ to conclude

$$
\left|K^{\circ}\right| \geq\left|\left(B_{\infty}^{2}\right)^{\circ}\right|=\left|B_{1}^{2}\right|
$$

In particular, we recover (1.3) on the plane, i.e.,

$$
|K|\left|K^{\circ}\right| \geq\left|B_{\infty}^{2}\right|\left|B_{1}^{2}\right|
$$

Proof of Theorem 1.2. First consider without loss of generality $K$ such that $|K|=$ $\left|B_{\infty}^{2}\right|$. Notice for $1 / p+1 / q=1,(1.9)$ can be compare in matrix form with

$$
Z_{p, N}(K)=\frac{1}{N^{1 / p}}[\mathbf{X}] B_{q}^{N} \quad \text { and } \quad Z_{p, N}\left(B_{\infty}^{2}\right)=\frac{1}{N^{1 / p}}[\mathbf{Y}] B_{q}^{N}
$$

Then, by Lemma 2.1 with $C=B_{q}^{N}$ one has

$$
\frac{1}{|K|^{p}} \mathbb{E}\left|\left([\mathbf{X}] B_{q}^{N}+r B_{2}^{n}\right)^{\circ}\right|^{-p} \leq \frac{1}{\left|B_{\infty}^{2}\right|^{p}} \mathbb{E}\left|\left([\widetilde{\mathbf{Y}}] B_{q}^{N}+r B_{2}^{n}\right)^{\circ}\right|^{-p},
$$

so it follows that

$$
\mathbb{E}\left|\left(\frac{1}{N^{1 / p}}[\mathbf{X}] B_{q}^{N}+r B_{2}^{n}\right)^{\circ}\right|^{-1} \leq \mathbb{E}\left|\left(\frac{1}{N^{1 / p}}[\mathbf{Y}] B_{q}^{N}+r B_{2}^{n}\right)^{\circ}\right|^{-1}
$$

which is (1.10). Moreover, taking limits on both sides of the expression above

$$
\lim _{N \rightarrow \infty} \mathbb{E}\left|\left(\frac{1}{N^{1 / p}}[\mathbf{X}] B_{q}^{N}+r B_{2}^{n}\right)^{\circ}\right|^{-1} \leq \lim _{N \rightarrow \infty} \mathbb{E}\left|\left(\frac{1}{N^{1 / p}}[\mathbf{Y}] B_{q}^{N}+r B_{2}^{n}\right)^{\circ}\right|^{-1}
$$

Inequality (1.11) follows from the continuity of the Lebesgue measure, the double application of the Dominated Convergence Theorem, and the almost sure convergence to the $p$-centroid body from the beginning of this section.

As we mentioned before, one can think of the Blaschke-Santaló inequality as the limit case $p=\infty$. We now show the limit case $p=1$ which we call the stochastic polar Bisztriczky-Böröczky inequality. 
Proof of Theorem 1.3. First consider without loss of generality $K$ such that $|K|=$ $\left|B_{\infty}^{2}\right|$. Notice we write

$$
Z_{N} K=\frac{1}{N}[\mathbf{X}] B_{\infty}^{N} \quad \text { and } \quad Z_{N} B_{\infty}^{2}=\frac{1}{N}[\mathbf{Y}] B_{\infty}^{N}
$$

Then, by Lemma 2.1 with $C=B_{\infty}^{N}$ one has

$$
\frac{1}{|K|} \mathbb{E}\left|\left([\mathbf{X}] B_{\infty}^{N}+r B_{2}^{n}\right)^{\circ}\right|^{-1} \leq \frac{1}{\left|B_{\infty}^{2}\right|} \mathbb{E}\left|\left([\mathbf{Y}] B_{\infty}^{N}+r B_{2}^{n}\right)^{\circ}\right|^{-1}
$$

so it follows that

$$
\mathbb{E}\left|\left(\frac{1}{N}[\mathbf{X}] B_{\infty}^{N}+r B_{2}^{n}\right)^{\circ}\right|^{-1} \leq \mathbb{E}\left|\left(\frac{1}{N}[\mathbf{Y}] B_{\infty}^{N}+r B_{2}^{n}\right)^{\circ}\right|^{-1}
$$

which is (1.12). Moreover, taking limits on both sides of the expression above

$$
\lim _{N \rightarrow \infty} \mathbb{E}\left|\left(\frac{1}{N}[\mathbf{X}] B_{\infty}^{N}+r B_{2}^{n}\right)^{\circ}\right|^{-1} \leq \lim _{N \rightarrow \infty} \mathbb{E}\left|\left(\frac{1}{N}[\mathbf{Y}] B_{\infty}^{N}+r B_{2}^{n}\right)^{\circ}\right|^{-1}
$$

Inequality (1.13) follows from the continuity of the Lebesgue measure, the double application of the Dominated Convergence Theorem, and the almost sure convergence to the centroid body from the beginning of this section.

Remark 2.2. Using (2.1) for $p=1$ and (2.3), one can determine the volume of the polar centroid body of $B_{\infty}^{2}$

$$
\begin{aligned}
\left|\left(Z B_{\infty}^{2}\right)^{\circ}\right| & =8 \int_{S}\left(\int_{B_{\infty}^{2}}|\langle x, z\rangle| d x\right)^{-2} d z \\
& =32 \int_{0}^{\pi / 2}\left(\int_{-1}^{1} \int_{-1}^{1}|x \cos \theta+y \sin \theta| d x d y\right)^{-2} d \theta \\
& =\frac{4 \pi}{\sqrt{3}}+6 .
\end{aligned}
$$

Therefore, using (2.7) and (1.13) we can give an estimate for the planar Centroid volume product:

$$
\left|(Z K)^{\circ}\right||K| \geq \frac{16 \pi}{\sqrt{3}}+24
$$


Lastly, to finish the section we first show in Lemma 2.3 that triangles are the maximizers for functional (2.6) which allows us to give a stochastic Mahler's Theorem on the plane for non-symmetric convex bodies.

Lemma 2.3. Let $T$ be a triangle, $N \geq 3, C=\operatorname{conv}\left\{e_{1}, \ldots, e_{N}\right\}$, and $p \geq 1$. Then

$$
W_{p, r}^{s \circ}(K ; N ; C) \leq W_{p, r}^{s \circ}(T ; N ; C)
$$

for all $K \in \mathcal{K}_{0}^{N}$.

Proof. Let $m \geq 3, K$ a polygon with $\left\{a_{1}, \ldots, a_{m}\right\}$ ordered clockwise vertices, $\theta$ a direction parallel to the line joining $a_{1}$ and $a_{3}$, and set

$$
K_{t}:=\operatorname{conv}\left\{a_{1}, a_{2}+t \theta, a_{3}, \ldots, a_{m}\right\}
$$

Then, there exists $\delta_{1}, \delta_{2}>0$ such that for $t \in\left[-\delta_{1}, \delta_{2}\right], K_{t}$ is a Rogers-Shephardmovement where $K_{-\delta_{1}}$ and $K_{\delta_{2}}$ have $m-1$ vertices. As $W_{p, r}^{s o}(K ; N ; C)$ is a convex functional, Theorem 2.4,

$$
W_{p, r}^{s \circ}(K ; N ; C) \leq \max \left\{W_{p, r}^{s \circ}\left(K_{-\delta_{1}} ; N ; C\right), W_{p, r}^{s \circ}\left(K_{\delta_{2}} ; N ; C\right)\right\}
$$

and iterating the above procedure one has

$$
W_{p, r}^{s \circ}(K ; N ; C) \leq W_{p, r}^{s \circ}(T ; N ; C) .
$$

The result follows by the invariance under linear transformations and the continuity of the functional.

Proof of Theorem 1.4. Let $e_{1}, \ldots, e_{N}$ be the standard orthonormal basis in $\mathbb{R}^{n}$, and $\tilde{C}=\operatorname{conv}\left\{e_{1}, \ldots, e_{N}\right\}$. Consider without loss of generality $K$ such that $|K|=$ $|T|=1$, then we are able to write

$$
[K]_{N}=[\mathbf{X}] \tilde{C} \quad \text { and } \quad\left[B_{\infty}^{2}\right]_{N}=[\tilde{\mathbf{Y}}] \tilde{C} .
$$


By Lemma 2.3 with $C=\tilde{C}$ we have

$$
\frac{1}{|K|^{p}} \mathbb{E}\left|\left([\mathbf{X}] \tilde{C}+r B_{2}^{n}\right)^{s \circ}\right|^{-p} \leq \frac{1}{\left|B_{\infty}^{2}\right|^{p}} \mathbb{E}\left|\left([\tilde{\mathbf{Y}}] \tilde{C}+r B_{2}^{n}\right)^{s \circ}\right|^{-p}
$$

and inequality (1.14) follows.

Remark 2.3. From (1.14) one has

$$
\lim _{N \rightarrow \infty} \mathbb{E}\left|\left([\mathbf{X}] \tilde{C}+r B_{2}^{n}\right)^{s \circ}\right|^{-1} \leq \lim _{N \rightarrow \infty} \mathbb{E}\left|\left([\tilde{\mathbf{Y}}] \tilde{C}+r B_{2}^{n}\right)^{s \circ}\right|^{-1}
$$

Thus, by the continuity of the Lebesgue measure and Dominated Convergence Theorem

$$
\mathbb{E}\left|\left(\lim _{N \rightarrow \infty}[\mathbf{X}] \tilde{C}+r B_{2}^{n}\right)^{s \circ}\right|^{-1} \leq \mathbb{E}\left|\left(\lim _{N \rightarrow \infty}[\tilde{\mathbf{Y}}] \tilde{C}+r B_{2}^{n}\right)^{s \circ}\right|^{-1}
$$

Therefore by Lemma 2.2 one has the almost sure convergence mentioned above. It follows that

$$
\left|\left(K+r B_{2}^{n}\right)^{s \circ}\right| \geq\left|\left(T+r B_{2}^{n}\right)^{s o}\right| .
$$

for each $r>0$. Hence we can appeal to dominated convergence once more and let $r \rightarrow 0$ to conclude

$$
\left|K^{s \circ}\right| \geq\left|T^{s \circ}\right|=\frac{27}{4},
$$

which recovers (1.3). 


\section{Chapter 3}

\section{A Stochastic Prékopa-Leindler Inequality for Log-Concave}

\section{Functions}

In this chapter we show that a "local" stochastic dominance underlies functional inequalities. We focus on the Prékopa-Leindler inequality for log-concave functions $f$, i.e., when $\log f$ is concave. We establish stochastic dominance inequalities for integrals of families of associated random functions.

\subsection{Definitions and notation}

We will denote by $\left\{e_{1}, \ldots, e_{n}\right\}$ the standard basis in $\mathbb{R}^{n}$. We recall that the Steiner symmetral of a non-empty compact set $A \subseteq \mathbb{R}^{n}$ with respect to $\theta^{\perp}, S_{\theta^{\perp}} A$, is the set with the property that for each line $l$ orthogonal to $\theta^{\perp}$ and meeting $A$, the set $l \cap S_{\theta^{\perp}} A$ is a closed segment with midpoint on $\theta^{\perp}$ and length equal to that of the set $l \cap A$. The mapping $S_{\theta^{\perp}}: A \rightarrow S_{\theta^{\perp}} A$ is called the Steiner symmetrization of $A$ with respect to $\theta^{\perp}$. In particular, if $K$ is a convex body

$$
S_{\theta^{\perp}} K=\left\{x+\lambda \theta: x \in P K,-\frac{u_{K}(x)-\ell_{K}(x)}{2} \leq \lambda \leq \frac{u_{K}(x)-\ell_{K}(x)}{2}\right\} .
$$


This shows that $S_{\theta^{\perp}} K$ is convex, since the function $u_{K}-\ell_{K}$ is concave. Moreover, $S_{\theta^{\perp}} K$ is symmetric with respect to $\theta^{\perp}$, it is closed, and by Fubini's theorem it has the same volume as $K$.

Let $A \subseteq \mathbb{R}^{n}$ be a Borel set with finite Lebesgue measure. The symmetric rearrangement, $A^{*}$, of $A$ is the open ball with center at the origin whose volume is equal to the measure of $A$. Since we choose $A^{*}$ to be open, $\mathbb{1}_{A^{*}}$ is lower semicontinuous. The symmetric decreasing rearrangement of $\mathbb{1}_{A}$ is defined by $\mathbb{1}_{A}^{*}=\mathbb{1}_{A^{*}}$. We say a Borel measurable function $f: \mathbb{R}^{n} \rightarrow[0, \infty)$ vanishes at infinity if for every $t>0$, the set $\left\{x \in \mathbb{R}^{n}: f(x)>t\right\}$ has finite Lebesgue measure. In such a case, the symmetric decreasing rearrangement $f^{*}$ is defined by

$$
f^{*}(x)=\int_{0}^{\infty} \mathbb{1}_{\{f>t\}}^{*}(x) d t=\int_{0}^{\infty} \mathbb{1}_{\{f>t\}^{*}}(x) d t .
$$

Observe that $f^{*}$ is radially symmetric, radially decreasing, and equimeasurable with $f$, i.e., $\{f>t\}$ and $\left\{f^{*}>t\right\}$ have the same measure for each $t>0$. Let $\left\{e_{1}, \ldots, e_{n}\right\}$ be an orthonormal basis of $\mathbb{R}^{n}$ such that $e_{1}=\theta$. Then, for $f$ vanishing at infinity, the Steiner symmetral $f(\cdot \mid \theta)$ of $f$ with respect to $\theta^{\perp}$ is defined as follows: set $f_{\left(x_{2}, \ldots, x_{n}\right), \theta}(t)=f\left(t, x_{2}, \ldots, x_{n}\right)$ and define $f^{*}\left(t, x_{2}, \ldots, x_{n} \mid \theta\right):=$ $\left(f_{\left(x_{2}, \ldots, x_{n}\right), \theta}\right)^{*}(t)$. In other words, we obtain $f^{*}(\cdot \mid \theta)$ by rearranging $f$ along every line parallel to $\theta$. We refer to the books $[52,90]$ or the introductory notes [17] for further background material on rearrangement of functions.

\subsection{Approximation of log-concave functions}

We start by recalling an approach to derving integral inequalities for functions by using certain higher-dimensional bodies of revolution. This method was used by Artstein, Klartag and Milman in [4] to extend Ball's functional Blaschke-Santaló inequality [6]; see $[7,33]$ for further developments. Such bodies were also used in the first derivation of the functional affine isoperimetric inequality [5]; see also [20]. As we mentioned in the introduction, Klartag [46] used the method to prove 
the Prékopa-Leindler inequality and we will review several key points for later use.

A function $f: \mathbb{R}^{n} \rightarrow[0, \infty)$ is called log-concave if $\log f$ is concave on its support. We also use the notion of s-concavity as in [12] meaning that $f$ is $s$ concave if $f^{s}$ is concave on its support; this differs from other uses of this term $[4,46,74]$. Any $s$-concave function, for $s>0$, is also log-concave. A useful approximation of a log-concave function $f$ by $\frac{1}{s}$-concave functions $f_{s}$ is given by

$$
f_{s}(x)=\left(1+\frac{\log f(x)}{s}\right)_{+}^{s},
$$

where $x_{+}=\max \{x, 0\}$. With this choice, $f_{s} \leq f$ for all $s>0$, and since a logconcave function is continuous on its support one has $f_{s} \rightarrow f$ locally uniformly on $\mathbb{R}^{n}$ as $s \rightarrow \infty$.

Let $\left(e_{1}, \ldots, e_{n+s}\right)$ be an orthonormal basis of $\mathbb{R}^{n+s}=\mathbb{R}^{n} \times \mathbb{R}^{s}$. For a measurable function $f: \mathbb{R}^{n} \rightarrow[0, \infty)$, we can associate the set

$$
\mathcal{K}_{f}^{s}:=\left\{(x, y) \in \mathbb{R}^{n} \times \mathbb{R}^{s}: x \in \overline{\operatorname{supp} f},|y| \leq f^{1 / s}(x)\right\}
$$

where $|\cdot|$ denotes the usual Euclidean norm. With this terminology, Brunn's principle means that a function on $\mathbb{R}^{n}$ is $\frac{1}{s}$-concave if and only if it is a marginal of the uniform measure on a convex body in $\mathbb{R}^{n+s}$. Thus $\mathcal{K}_{f}^{s}$ is convex if and only if $f$ is $\frac{1}{s}$-concave. Moreover,

$$
f(x)=\kappa_{s}^{-1} \int_{\mathbb{R}^{s}} \mathbb{1}_{\mathcal{K}_{f}^{s}}(x, y) d y
$$

where $\kappa_{s}$ is the volume of the $s$-dimensional Euclidean ball and hence

$$
\int_{\mathbb{R}^{n}} f(x) d x=\kappa_{s}^{-1}\left|\mathcal{K}_{f}^{s}\right|
$$

We also recall the notion of homothety of bodies, the $s$-Minkowski sum of two non-negative functions on $\mathbb{R}^{n}$, and their relation with the set (3.3). Let $\lambda>0$. 
We define the function $\lambda \cdot s f: \mathbb{R}^{n} \rightarrow[0, \infty)$ to be

$$
[\lambda \cdot s](x)=\lambda^{s} f\left(\frac{x}{\lambda}\right)
$$

This way $\mathcal{K}_{\lambda \cdot_{s} f}^{s}=\lambda \mathcal{K}_{f}^{s}$ so $\lambda \cdot{ }_{s} f$ is a functional analog of homothety of bodies, where if $f$ is an $\frac{1}{s}$-concave function, so is $\lambda{ }_{s} f$. Define also the $s$-Minkowski sum of two functions $f, g: \mathbb{R}^{n} \rightarrow[0, \infty)$ as

$$
\left[f \oplus_{s} g\right](v)=\sup \left\{\left(f(x)^{\frac{1}{s}}+g(y)^{\frac{1}{s}}\right)^{s}: v=x+y, x \in \operatorname{supp}(f), y \in \operatorname{supp}(g)\right\}
$$

whenever $v \in \operatorname{supp}(f)+\operatorname{supp}(g)$. If not, we set $\left[f \oplus_{s} g\right](v)=0$. This function is $\frac{1}{s}$-concave whenever $f$ and $g$ are, and

$$
\mathcal{K}_{f \oplus s}^{s}=\mathcal{K}_{f}^{s}+\mathcal{K}_{g}^{s}
$$

By (3.3) the latter body is convex when $f$ and $g$ are $\frac{1}{s}$-concave. Let us also denote

$$
\left(f \star_{\lambda, s} g\right)(v):=\left(\left(\lambda \cdot_{s} f\right) \oplus_{s}((1-\lambda) \cdot s g)\right)(v),
$$

and, as in the introduction,

$$
\left(f \star_{\lambda} g\right)(v):=\sup \left\{f^{\lambda}(x) g^{1-\lambda}(y): v=\lambda x+(1-\lambda) y\right\} .
$$

Therefore it follows that

$$
\mathcal{K}_{f \star \lambda, s}^{s}=\mathcal{K}_{\lambda \cdot s f}^{s}+\mathcal{K}_{(1-\lambda){ }_{s} g}^{s}=\mathcal{K}_{f}^{s}+{ }_{\lambda} \mathcal{K}_{g}^{s},
$$

where we have defined

$$
K+{ }_{\lambda} L:=\lambda K+(1-\lambda) L
$$

In this way, the Prékopa-Leindler inequality is derived in [46] as a "marginal" of the Brunn-Minkowski inequality in $\mathbb{R}^{n+s}$ when $s \rightarrow \infty$. We cannot directly 
apply the stochastic Brunn-Minkowski inequality (1.17) to the bodies $K=\mathcal{K}_{f}^{s}$ and $L=\mathcal{K}_{g}^{s}$ as this would involve different measures in each dimension $n+s$ and an increasing number of samples in each body. Instead, we revisit the proof of (1.17) and study operations beyond the convex hull of points that can be used in our stochastic approach. The following lemma provides a needed link. Henceforth, we denote the $s$-dimensional Euclidean ball centered at a point $x \in \mathbb{R}^{n}$ and $\rho \geq 0$ by

$$
B_{\rho}^{s}(x)=\left\{(x, \widehat{z}) \in \mathbb{R}^{n} \times \mathbb{R}^{s}:|\widehat{z}| \leq \rho\right\}
$$

Lemma 3.1. Let $w_{i}=\left(x_{i}, z_{i}\right) \in \mathbb{R}^{n} \times[0, \infty)$ for $i=1, \ldots, N$ and $s \in \mathbb{N}$. Let $T=T_{\left\{w_{i}\right\}, s}$ be the least $\frac{1}{s}$-concave function supported on $\operatorname{conv}\left\{x_{1}, \ldots, x_{N}\right\}$ such that $T\left(x_{i}\right) \geq z_{i}, i=1, \ldots, N$, i.e.,

$$
T(x)=\sup \left\{z^{s} \in \mathbb{R}:(x, z) \in \operatorname{conv}\left\{\left(x_{i}, r_{i}\right)\right\}_{i=1}^{N}\right\},
$$

where $r_{i}=z_{i}^{1 / s}, i=1, \ldots, N$. Then

$$
\mathcal{K}_{T}^{s}=\operatorname{conv}\left\{B_{r_{1}}^{s}\left(x_{1}\right), \ldots, B_{r_{N}}^{s}\left(x_{N}\right)\right\}
$$

Proof. For $x \in \operatorname{conv}\left\{x_{1}, \ldots, x_{N}\right\}$ and $\widehat{z} \in \mathbb{R}^{s}$ with $(x, \widehat{z}) \in \mathcal{K}_{T}^{s}$, there exists nonnegative $c_{1}, \ldots, c_{N}$ with $\sum_{i} c_{i}=1$ such that

$$
|\widehat{z}| \leq T(x)^{1 / s}=\sum_{i=1}^{N} c_{i} r_{i}
$$

and $x=\sum_{i=1}^{N} c_{i} x_{i}$. Thus, denoting $r:=\sum_{i=1}^{N} c_{i} r_{i}$, we have

$$
(x, \widehat{z}) \in B_{r}^{s}(x) \subseteq \operatorname{conv}\left\{B_{r_{1}}^{s}\left(x_{1}\right), \ldots, B_{r_{N}}^{s}\left(x_{N}\right)\right\}
$$

On the other hand, let $(x, \widehat{z}) \in \operatorname{conv}\left\{B_{r_{1}}^{s}\left(x_{1}\right), \ldots, B_{r_{N}}^{s}\left(x_{N}\right)\right\}$ so that for some $\left(c_{i}\right)$ with $c_{i} \geq 0, \sum_{i} c_{i}=1,(x, \widehat{z})=\sum_{i=1}^{N} c_{i}\left(x_{i}, \widehat{z}_{i}\right)$ where $\widehat{z}_{i} \in \mathbb{R}^{s}$ and $\left|\widehat{z}_{i}\right| \leq r_{i}$, for 
$i=1, \ldots, N$. Therefore,

$$
|\widehat{z}| \leq \sum_{i=1}^{N} c_{i} r_{i} \leq T(x)^{1 / s}
$$

so $(x, \widehat{z}) \in \mathcal{K}_{T}^{s}$ as desired.

\subsection{Convex hull and $\mathcal{M}$-addition operations}

Let $C \subseteq \mathbb{R}^{N}$ be a compact convex set; for $x_{1}, \ldots, x_{N} \in \mathbb{R}^{n}$, we view the $n \times N$ matrix $\left[x_{1}, \ldots, x_{N}\right]$ as an operator from $\mathbb{R}^{N}$ to $\mathbb{R}^{n}$. Then

$$
\left[x_{1}, \ldots, x_{N}\right] C=\left\{\sum_{i} c_{i} x_{i}: c=\left(c_{i}\right) \in C\right\}
$$

produces a convex set in $\mathbb{R}^{n}$. This viewpoint was used by the Paouris and Pivovarov in [68] in randomized isoperimetric inequalities for convex sets; for the special case $C=\operatorname{conv}\left\{e_{1}, \ldots, e_{N}\right\}$, one has

$$
\left[x_{1}, \ldots, x_{N}\right] C=\operatorname{conv}\left\{x_{1}, \ldots, x_{N}\right\}
$$

Moreover, for vectors $x_{1} \ldots, x_{N}, x_{N+1}, \ldots, x_{N+M}$, we have

$$
\begin{aligned}
& {\left[x_{1}, \ldots, x_{N}\right] C_{N}+\left[x_{N+1}, \ldots, X_{N+M}\right] C_{M}} \\
& \quad=\left[x_{1}, \ldots, x_{N}\right] C_{N}+\left[x_{N+1}, \ldots, x_{N+M}\right] C_{M} \\
& \quad=\left[x_{1}, \ldots, x_{N+M}\right]\left(C_{N}+\widehat{C}_{M}\right),
\end{aligned}
$$

where $C_{k}=\operatorname{conv}\left\{e_{1}, \ldots, e_{k}\right\}$ for $k=N, M$ and $\widehat{C}_{M}=\operatorname{conv}\left\{e_{N+1}, \ldots, e_{N+M}\right\}$. The convex operations on points (3.9) can also be generalized to convex operations on sets by using the notion of $\mathcal{M}$-addition. This was introduced by Gardner, Hug, and Weil $[36,37]$ as a unifying framework for operations in Lutwak, Yang, and Zhang's $L_{p}$ and Orlicz Brunn-Minkowski theory (see e.g., [55]). For $\mathcal{M} \subseteq \mathbb{R}^{N}$ and 
subsets $K_{1}, \ldots, K_{N}$ in $\mathbb{R}^{n}$, their $\mathcal{M}$-combination is defined by

$$
\oplus_{\mathcal{M}}\left(K_{1}, \ldots, K_{N}\right)=\left\{\sum_{i=1}^{N} m_{i} x_{i}: x_{i} \in K_{i},\left(m_{1}, \ldots, m_{N}\right) \in \mathcal{M}\right\} .
$$

Thus, with this notation, for $C=\mathcal{M}$,

$$
\oplus_{C}\left(\left\{x_{1}\right\}, \ldots,\left\{x_{N}\right\}\right)=\left[x_{1}, \ldots, x_{N}\right] C
$$

Additionally, when $K_{1}, \ldots, K_{N}$ are convex and $\mathcal{M}$ is compact, convex and contained in the positive orthant or origin-symmetric, then $\oplus_{\mathcal{M}}\left(K_{1}, \ldots, K_{N}\right)$ is convex [36, Theorem 6.1].

To connect with the bodies defined in $\S 3.2$, we use $\mathcal{M}$-combinations of parallel $s$-dimensional Euclidean balls lying orthogonal to $\mathbb{R}^{n}$ in $\mathbb{R}^{n+s}$. Let $C \subset \mathbb{R}^{N}$ be a compact, convex set contained in the positive orthant, $\rho_{1}, \ldots, \rho_{N} \in[0, \infty)$ and $x_{1}, \ldots, x_{N} \in \mathbb{R}^{N}$. Then,

$$
\oplus_{C}\left(B_{\rho_{1}}^{s}\left(x_{1}\right), \ldots, B_{\rho_{N}}^{s}\left(x_{N}\right)\right)
$$

form the body of revolution of some $\frac{1}{s}$-concave function. By choosing $C=$ $\operatorname{conv}\left\{e_{1}, \ldots, e_{N}\right\}$, one simple takes the convex hull in $\mathbb{R}^{n+s}$ of the $s$-dimensional balls.

\subsection{Multiple integral rearrangement inequalities}

When an isoperimetric principle admits a proof by symmetrization, like (1.16) for example, it is often meaningful to instead carry out such symmetrization on a suitable (product) probability space. In this section, we show that the multiple integral rearrangement inequalities of Rogers [82], and Brascamp, Lieb, and Luttinger [16] interface well with our approach. In particular, Christ's version [24] of the latter inequalities is especially applicable; the following formulation is 
convenient for our purpose.

Theorem 3.1. Let $f_{1}, \ldots, f_{N}$ be non-negative integrable functions on $\mathbb{R}^{n}$ and $F$ : $\left(\mathbb{R}^{n}\right)^{N} \rightarrow[0, \infty)$. Then

$$
\begin{aligned}
& \int_{\left(\mathbb{R}^{n}\right)^{N}} F\left(x_{1}, \ldots, x_{N}\right) \prod_{i=1}^{N} f_{i}\left(x_{i}\right) d x_{1} \ldots d x_{N} \\
& \geq \int_{\left(\mathbb{R}^{n}\right)^{N}} F\left(x_{1}, \ldots, x_{N}\right) \prod_{i=1}^{N} f_{i}^{*}\left(x_{i}\right) d x_{1} \ldots d x_{N},
\end{aligned}
$$

whenever $F$ satisfies the following condition: for each $\theta \in \mathbb{S}^{n-1}$ and all $Y:=$ $\left\{y_{1}, \ldots, y_{N}\right\} \subseteq \theta^{\perp}$, the function $F_{Y}: \mathbb{R}^{N} \rightarrow[0, \infty)$ defined by

$$
F_{Y, \theta}\left(t_{1}, \ldots, t_{N}\right):=F\left(y_{1}+t_{1} \theta, \ldots, y_{N}+t_{N} \theta\right)
$$

is even and quasi-convex.

The condition on $F$, namely Steiner convexity, allows the theorem to be proved via iterated Steiner symmetrization; notice this terminology differs from the one in [24]. Of special interest, this condition interfaces well with shadow systems, e.g., $[21,81]$; see [69] for further background and references. For context, we recall only several examples before treating the functionals involved in our main theorems of the chapter.

A fundamental example of a Steiner convex function is the absolute value of the determinant, $F\left(x_{1}, \ldots, x_{n}\right)=\left|\operatorname{det}\left(\left[x_{1}, \ldots, x_{n}\right]\right)\right|($ when $N=n)$; this is the key property behind Busemann's random simplex inequality [18]. More generally, Groemer [41] showed that for $N>n$, the functional

$$
F\left(x_{1}, \ldots, x_{N}\right)=\left|\operatorname{conv}\left\{x_{1}, \ldots, x_{N}\right\}\right|
$$

is also Steiner convex. While the latter examples involve points $x_{i}$, analogous 
results hold for convex hulls of Euclidean balls

$$
B_{\rho_{i}}\left(x_{i}\right):=\left\{u \in \mathbb{R}^{n}:\left|u-x_{i}\right| \leq \rho_{i}\right\}
$$

Indeed, in [72], Pfiefer showed that for $\rho_{1}, \ldots, \rho_{N} \geq 0$, the functional

$$
F\left(x_{1}, \ldots, x_{N}\right)=\left|\operatorname{conv}\left\{B_{\rho_{1}}\left(x_{1}\right), \ldots, B_{\rho_{N}}\left(x_{N}\right)\right\}\right|
$$

satisfies the Steiner convexity property.

In [68], the functional in (3.12) was generalized to include operations beyond the convex hull preserving the Steiner convexity property. Namely, let $C \subseteq \mathbb{R}^{N}$ be a compact convex set contained in the positive orthant and $x_{1}, \ldots, x_{N}$, then

$$
F\left(x_{1}, \ldots, x_{N}\right)=\left|\left[x_{1}, \ldots, x_{N}\right] C\right|
$$

is Steiner convex. As noted in [69], convex operations on points in (3.14) can be combined with Euclidean balls (as in (3.13)) by using the notion of $\mathcal{M}$-addition defined in $\S 3.3$. We show the functional connected to the $\mathcal{M}$-addition of parallel $s$-dimensional balls in $\mathbb{R}^{n+s}(3.11)$ is Steiner convex.

Proposition 3.1. Let $\rho_{1}, \ldots, \rho_{N} \in[0, \infty)$ and $C$ a convex set contained in the positive orthant. Then the function $F:\left(\mathbb{R}^{n}\right)^{N} \rightarrow[0, \infty)$ defined by

$$
F\left(x_{1}, \ldots, x_{N}\right)=\left|\oplus_{C}\left(B_{\rho_{1}}^{s}\left(x_{1}\right), \ldots, B_{\rho_{N}}^{s}\left(x_{N}\right)\right)\right|
$$

is Steiner convex.

Proof. We suppose without loss of generality that $\theta=e_{1}$, where $\left\{e_{1}, \ldots, e_{n}\right\}$ denotes the standard basis in $\mathbb{R}^{n}$. Let $Y=\left\{y_{1}, \ldots, y_{N}\right\} \subseteq \theta^{\perp}$. We define the 
function $F_{Y}: \mathbb{R}^{N} \rightarrow[0, \infty)$ by

$$
\begin{aligned}
F_{Y}(t) & =\mid \oplus_{C}\left(B_{\rho_{1}}^{s}\left(\left(t_{1}, y_{1}\right)\right), \ldots, B_{\rho_{N}}^{s}\left(\left(t_{N}, y_{N}\right)\right) \mid\right. \\
& =\left|\left\{\sum_{i=1}^{N} c_{i}\left(t_{i}, y_{i}, \widehat{z}_{i}\right):\left|\widehat{z}_{i}\right| \leq \rho_{i}\right\}\right| .
\end{aligned}
$$

To check that $F_{Y}$ is even we note that the sets involved in the expressions for $F_{Y}(t)$ and $F_{Y}(-t)$ are reflections of each other about $\theta^{\perp}$ and hence have equal volume.

To prove convexity, let $r, t \in \mathbb{R}^{N}$. For $a=\left(a_{i}\right)$ in $\left\{r, t, \frac{r}{2}+\frac{t}{2}\right\}$, we write

$$
\oplus_{C}\left(\left\{B_{\rho_{i}}^{s}\left(a_{i}, y_{i}\right)\right\}\right):=\oplus_{C}\left(B_{\rho_{1}}^{s}\left(\left(a_{1}, y_{1}\right)\right), \ldots, B_{\rho_{N}}^{s}\left(\left(a_{N}, y_{N}\right)\right)\right)
$$

Let $P: \mathbb{R}^{n+s} \rightarrow \theta^{\perp} \times \mathbb{R}^{s}$ denote the orthogonal projection. Note that

$$
D:=P\left(\oplus_{C}\left(\left\{B_{\rho_{i}}^{s}\left(a_{i}, y_{i}\right)\right\}\right)\right)
$$

is independent of $a$. Thus we define as in $\S 2.1, u_{a}, \ell_{a}: D \rightarrow \mathbb{R}$ by

$$
u_{a}(v)=u\left(\oplus_{C}\left(\left\{B_{\rho_{i}}^{s}\left(a_{i}, y_{i}\right)\right\}\right), v\right)
$$

and

$$
\ell_{a}(v)=\ell\left(\oplus_{C}\left(\left\{B_{\rho_{i}}^{s}\left(a_{i}, y_{i}\right)\right\}\right), v\right)
$$

Next, we set $u=\frac{1}{2}\left(u_{r}+u_{t}\right)$ and $\ell=\frac{1}{2}\left(\ell_{r}+\ell_{t}\right)$ and define

$$
E:=\{(\lambda, v): v \in D, \ell(v) \leq \lambda \leq u(v)\}
$$

We claim that

$$
\oplus_{C}\left(\left\{B_{\rho_{i}}^{s}\left(\frac{r_{i}+t_{i}}{2}, y_{i}\right)\right\}\right) \subseteq E
$$


To see this, let $w \in \oplus_{C}\left(\left\{B_{\rho_{i}}^{s}\left(\frac{r_{i}+t_{i}}{2}, y_{i}\right)\right\}\right)$ so that for some $c \in C$,

$$
\sum_{i=1}^{N} c_{i}\left(\frac{r_{i}+t_{i}}{2}, y_{i}, \widehat{z}_{i}\right)=\left(\sum_{i=1}^{N} c_{i}\left(\frac{r_{i}+t_{i}}{2}\right), y, \widehat{z}\right)
$$

where $y=\sum_{i} c_{i} y_{i}$ and $\widehat{z}=\sum_{i} c_{i} \widehat{z}_{i}$ Thus for $a \in\{r, t\}$, we have

$$
\sum_{i=1}^{N} c_{i}\left(a_{i}, y_{i}, \widehat{z}_{i}\right)=\left(\sum_{i=1}^{N} c_{i} a_{i}, y, \widehat{z}\right) \in \oplus_{C}\left(\left\{B_{\rho_{i}}^{s}\left(a_{i}, y_{i}\right)\right\}\right)
$$

hence $\ell_{a}(0, y, \widehat{z}) \leq \sum_{i=1}^{N} c_{i} a_{i} \leq u_{a}(0, y, \widehat{z})$. Thus,

$$
\ell(0, y, \widehat{z}) \leq \frac{1}{2} \sum_{i=1}^{N} c_{i} r_{i}+\frac{1}{2} \sum_{i=1}^{N} c_{i} t_{i} \leq u(0, y, \widehat{z})
$$

which shows that $w \in E$ and establishes (3.16). Hence

$$
\begin{aligned}
|E| & =\int_{D}(u(v)-\ell(v)) d v \\
& =\frac{1}{2} \int_{D}\left(u_{r}-\ell_{r}\right)(v) d v+\frac{1}{2} \int_{D}\left(u_{t}-\ell_{t}\right)(v) d v \\
& =\frac{1}{2}\left|\oplus_{C}\left(\left\{B_{\rho_{i}}^{s}\left(r_{i}, y_{i}\right)\right\}\right)\right|+\frac{1}{2}\left|\oplus_{C}\left(\left\{B_{\rho_{i}}^{s}\left(t_{i}, y_{i}\right)\right\}\right)\right|
\end{aligned}
$$

which completes the proof.

\subsection{Proofs of stochastic Prékopa-Leindler and Groemer inequalities}

Proof of Theorem 1.6. For $\left(x_{1}, z_{1}\right), \ldots,\left(x_{N}, z_{N}\right) \in \mathbb{R}^{n} \times[0, \infty)$, write $w_{i}=\left(x_{i}, z_{i}\right)$ and let $T_{\left\{w_{i}\right\}}$ be the least log-concave function supported on conv $\left\{x_{1}, \ldots, x_{N}\right\}$ with $T\left(x_{i}\right) \geq z_{i}, i=1, \ldots, N$, i.e.,

$$
T_{\left\{w_{i}\right\}}(x)=\exp \left(\sup \left\{z:(x, z) \in \operatorname{conv}\left\{\left(x_{1}, \log z_{1}\right), \ldots,\left(x_{N}, \log z_{N}\right)\right\}\right)\right.
$$


With this notation, we set

$$
F\left(w_{1}, \ldots, w_{N}\right)=\int_{\mathbb{R}^{n}} T_{\left\{w_{i}\right\}}(x) d x
$$

Let $f: \mathbb{R}^{n} \rightarrow[0, \infty)$ be an integrable log-concave function. Sample independent random vectors $W_{i}=\left(X_{i}, Z_{i}\right), i=1, \ldots, N$, according to the uniform Lebesgue measure on $G_{f}$ (cf. (1.22)). Then the random function $[f]_{N}$ defined as in (1.24) satisfies

$$
\begin{aligned}
\mathbb{P}\left(\int_{\mathbb{R}^{n}}[f]_{N}(x) d x>\alpha\right) & =\mathbb{E} \mathbb{1}_{\{F>\alpha\}}\left(W_{1}, \ldots, W_{N}\right) \\
& =\frac{1}{\|f\|_{1}^{N}} \int_{N} \mathbb{1}_{\{F>\alpha\}}(\bar{w}) \prod_{i=1}^{N} \mathbb{1}_{\left[0, f\left(x_{i}\right)\right]}\left(z_{i}\right) d \bar{w}
\end{aligned}
$$

where $\int_{N}$ denotes the integral on $\left(\mathbb{R}^{n} \times[0, \infty)\right)^{N}$ and

$$
\bar{w}=\left(w_{1}, \ldots, w_{N}\right), \quad d \bar{w}=d w_{1} \ldots d w_{N}
$$

It is sufficient to prove the theorem for $f_{\varepsilon}(x):=f(x) \mathbb{1}_{\{f>\varepsilon\}}(x)$ for each fixed $\varepsilon>0$. To see this, note that $\left(f_{\varepsilon}\right)^{*}=\left(f^{*}\right)_{\varepsilon}$. Thus if $W_{i}^{\varepsilon}=\left(X_{i}^{\varepsilon}, Z_{i}^{\varepsilon}\right), i=1, \ldots, N$ are independent random vectors distributed according to the uniform Lebesgue measure on $G_{f_{\varepsilon}}$, we have

$$
\begin{aligned}
\mathbb{P}\left(\int_{\mathbb{R}^{n}}\left[f_{\varepsilon}\right]_{N}(x) d x>\alpha\right) & =\mathbb{E} \mathbb{1}_{\{F>\alpha\}}\left(W_{1}^{\varepsilon}, \ldots, W_{N}^{\varepsilon}\right) \\
& =\frac{1}{\left\|f_{\epsilon}\right\|_{1}^{N}} \int_{N} \mathbb{1}_{\{F\}>\alpha\}}(\bar{w}) \prod_{i=1}^{N} \mathbb{1}_{\left[\varepsilon, f\left(x_{i}\right)\right]}\left(z_{i}\right) d \bar{w}
\end{aligned}
$$

Since $\left\|f_{\varepsilon}\right\|_{1} \rightarrow\|f\|_{1}$ and $f_{\varepsilon} \leq f$, dominated convergence implies

$$
\mathbb{P}\left(\int_{\mathbb{R}^{n}}[f]_{N}(x) d x>\alpha\right)=\lim _{\epsilon \rightarrow 0} \mathbb{P}\left(\int_{\mathbb{R}^{n}}\left[f_{\varepsilon}\right]_{N}(x) d x>\alpha\right) .
$$


For $s>0$ and $x \in \mathbb{R}^{n}$, we define

$$
\left[f_{\epsilon}\right]_{N, s}(x)=\left(1+\frac{\log \left[f_{\epsilon}\right]_{N}(x)}{s}\right)_{+}^{s} .
$$

For every $x$, we have almost sure convergence $\left[f_{\varepsilon}\right]_{N, s}(x) \rightarrow\left[f_{\varepsilon}\right]_{N}(x)$ as $s \rightarrow \infty$. Since $\left[f_{\varepsilon}\right]_{N, s},\left[f_{\varepsilon}\right]_{N}$ are dominated by $f$, this implies almost sure convergence of the integrals

$$
\int_{\mathbb{R}^{n}}\left[f_{\varepsilon}\right]_{N}(x) d x=\lim _{s \rightarrow 0} \int_{\mathbb{R}^{n}}\left[f_{\varepsilon}\right]_{N, s}(x) d x
$$

The latter implies that

$$
\mathbb{P}\left(\int_{\mathbb{R}^{n}}\left[f_{\varepsilon}\right]_{N}(x) d x \leq \alpha\right)=\lim _{s \rightarrow \infty} \mathbb{P}\left(\int_{\mathbb{R}^{n}}\left[f_{\varepsilon}\right]_{N, s}(x) d x \leq \alpha\right)
$$

at all continuity points of

$$
[0, \infty) \ni a \mapsto \mathbb{P}\left(\int_{\mathbb{R}^{n}}\left[f_{\varepsilon}\right]_{N}(x) d x \leq a\right)
$$

However, since $N>n+1$ and $f_{\varepsilon}$ is absolutely continuous with respect to Lebesgue measure, the random variable $\int_{\mathbb{R}^{n}}\left[f_{\varepsilon}\right]_{N}(x) d x$ is positive almost surely and (3.21) is continuous on $[0, \infty)$.

Thus it is sufficient to prove the theorem for $\left[f_{\varepsilon}\right]_{N, s}$ with $s$ large enough. For $s>-\log \epsilon$, we have $R_{i}:=R_{i}\left(Z_{i}^{\varepsilon}\right):=\left(1+\frac{\log Z_{i}^{\varepsilon}}{s}\right)>0$ for all $i \in\{1, \ldots, N\}$. Then

$$
\left[f_{\epsilon}\right]_{N, s}(x)=\sup \left\{z^{s} \in \mathbb{R}:(x, z) \in H_{f_{\epsilon}, N, s}\right\},
$$

where

$$
H_{f_{\epsilon}, N, s}:=\operatorname{conv}\left\{\left(X_{1}^{\varepsilon}, R_{1}\left(Z_{1}^{\varepsilon}\right)\right), \ldots,\left(X_{N}^{\varepsilon}, R_{N}\left(Z_{N}^{\varepsilon}\right)\right)\right\} .
$$

By Lemma 3.1,

$$
\mathcal{K}_{\left[f_{\epsilon}\right]_{N, s}}^{s}=\operatorname{conv}\left\{B_{R_{1}}^{s}\left(X_{1}^{\varepsilon}\right), \ldots, B_{R_{N}}^{s}\left(X_{N}^{\varepsilon}\right)\right\}
$$


By (3.4),

$$
\int_{\mathbb{R}^{n}}\left[f_{\varepsilon}\right]_{N, s}(x) d x=\kappa_{s}^{-1}\left|\mathcal{K}_{\left[f_{\varepsilon}\right]_{N, s}}^{s}\right|
$$

For $z_{1}, \ldots, z_{N} \in[0, \infty)$, we write $\rho_{i}=\rho_{i}\left(z_{i}\right)=\left(1+\frac{\log z_{i}}{s}\right)$ and define

$$
F_{s}\left(\left(x_{1}, z_{1}\right), \ldots,\left(x_{N}, z_{N}\right)\right)=\left|\operatorname{conv}\left\{B_{\rho_{1}}^{s}\left(x_{1}\right), \ldots, B_{\rho_{N}}^{s}\left(x_{N}\right)\right\}\right|
$$

Then for each fixed $z_{1}, \ldots, z_{N}$,

$$
\left(x_{1}, \ldots, x_{N}\right) \mapsto F_{s}\left(\left(x_{1}, z_{1}\right), \ldots,\left(x_{N}, z_{N}\right)\right)
$$

is a Steiner convex function by Proposition 3.1. Applying Fubini, writing $d \bar{w}=$ $d \bar{x} d \bar{z}$ and invoking Theorem 3.1, we obtain

$$
\begin{aligned}
\mathbb{P}\left(\left|\mathcal{K}_{\left[f_{\varepsilon}\right]_{N, s}}^{s}\right|>\alpha\right) & =\frac{1}{\left\|f_{\varepsilon}\right\|_{1}^{N}} \int_{N} \mathbb{1}_{\left\{F_{s}>\alpha\right\}}(\bar{w}) \prod_{i=1}^{N} \mathbb{1}_{\left[\varepsilon, f\left(x_{i}\right)\right]}\left(z_{i}\right) d \bar{w} \\
& =\frac{1}{\left\|f_{\varepsilon}\right\|_{1}^{N}} \int_{[0, \infty)^{N}}\left(\int_{\left(\mathbb{R}^{n}\right)^{N}} \mathbb{1}_{\left\{F_{s}>\alpha\right\}} \prod_{i=1}^{N} \mathbb{1}_{\left[\varepsilon, f\left(x_{i}\right)\right]}\left(z_{i}\right) d \bar{x}\right) d \bar{z} \\
& \geq \frac{1}{\left\|f_{\varepsilon}^{*}\right\|_{1}^{N}} \int_{[0, \infty)^{N}}\left(\int_{\left(\mathbb{R}^{n}\right)^{N}} \mathbb{1}_{\left\{F_{s}>\alpha\right\}} \prod_{i=1}^{N} \mathbb{1}_{\left[\varepsilon, f^{*}\left(x_{i}\right)\right]}\left(z_{i}\right) d \bar{x}\right) d \bar{z} \\
& =\frac{1}{\left\|f_{\varepsilon}^{*}\right\|_{1}^{N}} \int_{N} \mathbb{1}_{\left\{F_{s}>\alpha\right\}}(\bar{w}) \prod_{i=1}^{N} \mathbb{1}_{\left[\varepsilon, f^{*}\left(x_{i}\right)\right]}\left(z_{i}\right) d \bar{w} \\
& =\mathbb{P}\left(\left|\mathcal{K}_{\left[f_{\varepsilon}^{*}\right]_{N, s}}^{s}\right|>\alpha\right) .
\end{aligned}
$$

The result now follows from (3.22) applied to $f_{\varepsilon}^{*}$.

Proof of Theorem 1.5. For $w_{i}=\left(x_{i}, z_{i}\right) \in \mathbb{R}^{n} \times \mathbb{R}^{s}, i=1, \ldots, M+N$, we set

$$
F\left(w_{1}, \ldots, w_{N+M}\right)=\int_{\mathbb{R}^{n}}\left(T_{\left\{w_{i}\right\}}^{(N)} \star_{\lambda} T_{\left\{w_{i}\right\}}^{(M)}\right)(v) d v
$$

where $T_{\left\{w_{i}\right\}}^{(N)}$ and $T_{\left\{w_{i}\right\}}^{(M)}$ are the least log-concave functions above the collections $\left\{w_{i}\right\}_{i \leq N}$ and $\left\{w_{i}\right\}_{N+1 \leq i \leq M}$, respectively (as defined as in (3.17)). Then the stochas- 
tic approximations $[f]_{N},[g]_{M}$ to $f, g$ satisfy

$$
\begin{aligned}
& \mathbb{P}\left(\int_{\mathbb{R}^{n}}\left([f]_{N} \star_{\lambda}[g]_{M}\right)(v) d v>\alpha\right) \\
& \quad=\frac{1}{\prod_{i=1}^{M+N}\left\|h_{i}\right\|_{1}} \int_{N+M} \mathbb{1}_{\{F>\alpha\}}(\bar{w}) \prod_{i=1}^{N+M} \mathbb{1}_{\left[0, h_{i}\left(x_{i}\right)\right]}\left(z_{i}\right) d \bar{w}
\end{aligned}
$$

where $\int_{N+M}$ is the integral on $\left(\mathbb{R}^{n} \times[0, \infty)\right)^{N+M}, \bar{w}$ and $d \bar{w}$ are as in $(3.18), h_{i}=f_{i}$ for $i=1, \ldots, N$ and $h_{i}=g_{i}$ for $i=N+1, \ldots, N+M$. For $\varepsilon>0$, we apply the latter identity with $f_{\varepsilon}$ and $g_{\varepsilon}$ and use dominated convergence to get

$$
\mathbb{P}\left(\int_{\mathbb{R}^{n}}\left([f]_{N} \star_{\lambda}[g]_{M}\right)(v) d v>\alpha\right)=\lim _{\varepsilon \rightarrow 0} \mathbb{P}\left(\int_{\mathbb{R}^{n}}\left(\left[f_{\epsilon}\right]_{N} \star_{\lambda}\left[g_{\epsilon}\right]_{M}\right)(v) d v>\alpha\right) .
$$

For $\varepsilon>0$, we sample independent random vectors $\left\{\left(X_{i}^{\varepsilon}, Z_{i}^{\varepsilon}\right)\right\}_{i=1}^{N}$ uniformly in $G_{f_{\varepsilon}}$ and $\left\{\left(X_{i}^{\varepsilon}, Z_{i}^{\varepsilon}\right)\right\}_{i=N+1}^{N+M}$ uniformly in $G_{g_{\varepsilon}}$. For $s>-\log \varepsilon$, we define $\left[f_{\epsilon}\right]_{N, s}$, and $\left[g_{\epsilon}\right]_{M, s}$ as in (3.19). Note that for each $v \in \mathbb{R}^{n}$, we have almost sure convergence

$$
\left(\left[f_{\epsilon}\right]_{N} \star_{\lambda}\left[g_{\epsilon}\right]_{M}\right)(v)=\lim _{s \rightarrow \infty}\left(\left[f_{\epsilon}\right]_{N, s} \star_{\lambda, s}\left[g_{\epsilon}\right]_{M, s}\right)(v) .
$$

Since $\left[f_{\epsilon}\right]_{N, s} \star_{\lambda, s}\left[g_{\epsilon}\right]_{M, s}$ and $\left[f_{\epsilon}\right]_{N} \star_{\lambda}\left[g_{\epsilon}\right]_{M}$ are dominated by $f \star_{\lambda} g$, we can argue as in the proof of Theorem 1.6 to get, for each $\alpha \geq 0$,

$$
\begin{aligned}
& \mathbb{P}\left(\int_{\mathbb{R}^{n}}\left(\left[f_{\epsilon}\right]_{N} \star_{\lambda}\left[g_{\epsilon}\right]_{M}\right)(v) d v \leq \alpha\right) \\
& \quad=\lim _{s \rightarrow \infty} \mathbb{P}\left(\int_{\mathbb{R}^{n}}\left(\left[f_{\epsilon}\right]_{N, s} \star_{\lambda, s}\left[g_{\epsilon}\right]_{M, s}\right)(v) d v \leq \alpha\right) .
\end{aligned}
$$

Since $\left[f_{\epsilon}\right]_{N, s} \star_{\lambda, s}\left[g_{\epsilon}\right]_{M, s}$ is $\frac{1}{s}$-concave, the body $\mathcal{K}_{\left[f_{\epsilon}\right]_{N, s} \star_{\lambda, s}\left[g_{\epsilon}\right]_{M, s}}$ is convex. Moreover, by (3.4),

$$
\int_{\mathbb{R}^{n}}\left(\left[f_{\epsilon}\right]_{N, s} \star_{\lambda, s}\left[g_{\epsilon}\right]_{M, s}\right)(v) d v=\kappa_{s}^{-1}\left|K_{\left[f_{\epsilon}\right]_{N, s} \star_{\lambda, s}\left[g_{\epsilon}\right]_{M, s}}\right| .
$$


Recalling (3.5), we have

$$
\begin{aligned}
\mathcal{K}_{\left[f_{\epsilon}\right]_{N, s} \star_{\lambda, s}\left[g_{\epsilon}\right]_{M, s}}^{s} & =\lambda \mathcal{K}_{\left[f_{\epsilon}\right]_{N, s}}^{s}+(1-\lambda) \mathcal{K}_{\left[g_{\varepsilon}\right]_{M, s}}^{s} \\
& =\mathcal{K}_{\left[f_{\epsilon}\right]_{N, s}}^{s}+{ }_{\lambda} \mathcal{K}_{\left[g_{\varepsilon}\right]_{M, s}}^{s} .
\end{aligned}
$$

Set $R_{i}:=R_{i}\left(Z_{i}\right):=\left(1+\frac{\log Z_{i}^{\varepsilon}}{s}\right)$. By Lemma 3.1, we have

$$
\begin{aligned}
\mathcal{K}_{\left[f_{\epsilon}\right]_{N, s}}^{s} & =\operatorname{conv}\left\{B_{R_{1}}^{s}\left(X_{1}\right), \ldots, B_{R_{N}}^{s}\left(X_{N}\right)\right\} \\
& =\oplus_{C_{N}}\left(\left\{B_{R_{i}}^{s}\left(X_{i}\right)\right\}_{i=1}^{N}\right)
\end{aligned}
$$

where $C_{N}=\operatorname{conv}\left\{e_{1}, \ldots, e_{N}\right\}$. Similarly,

$$
\begin{aligned}
\mathcal{K}_{\left[g_{\epsilon}\right]_{M, s}}^{s} & =\operatorname{conv}\left\{B_{R_{N+1}}^{s}\left(X_{N+1}\right), \ldots, B_{R_{N+M}}^{s}\left(X_{N+M}\right)\right\} \\
& =\oplus_{C_{M}}\left(\left\{B_{R_{i}}^{s}\left(X_{i}\right)\right\}_{i=N+1}^{M}\right),
\end{aligned}
$$

where $C_{M}=\operatorname{conv}\left\{e_{1}, \ldots, e_{M}\right\}$. Thus if we write $\widehat{C}_{M}=\operatorname{conv}\left\{e_{N+1}, \ldots, e_{N+M}\right\}$, (which is similar to (3.10)), we have

$$
\begin{aligned}
\mathcal{K}_{\left[f_{\epsilon}\right]_{N, s} \star_{\lambda, s}\left[g_{\epsilon}\right]_{M, s}}^{s} & =\oplus_{C_{N}}\left(\left\{B_{R_{i}}^{s}\left(X_{i}\right)\right\}_{i=1}^{N}\right)+_{\lambda} \oplus_{C_{M}}\left(\left\{B_{R_{i}}^{s}\left(X_{i}\right)\right\}_{i=N+1}^{N+M}\right) \\
& =\oplus_{C_{N}+{ }_{\lambda} \widehat{C}_{M}}\left(\left\{B_{R_{i}}^{s}\left(X_{i}\right)\right\}_{i=1}^{N+M}\right) .
\end{aligned}
$$

For $z_{1}, \ldots, z_{N+M} \in[0, \infty)$, write $\rho_{i}=\rho_{i}\left(z_{i}\right)=\left(1+\frac{\log z_{i}}{s}\right)$ and define

$$
F_{s}\left(\left(x_{1}, z_{1}\right), \ldots,\left(x_{N+M}, z_{N+M}\right)\right)=\left|\oplus_{C_{N}+{ }_{\lambda} \widehat{C}_{M}}\left(\left\{B_{\rho_{i}}^{s}\left(x_{i}\right)\right\}_{i=1}^{N+M}\right)\right| .
$$

Then for each fixed $z_{1}, \ldots, z_{N+M}$, the function

$$
\left(x_{1}, \ldots, x_{N+M}\right) \mapsto F_{s}\left(\left(x_{1}, z_{1}\right), \ldots,\left(x_{N+M}, z_{N+M}\right)\right)
$$


is Steiner convex by Proposition 3.1. By Fubini and Theorem 3.1, we have

$$
\begin{aligned}
& \mathbb{P}\left(\left|\mathcal{K}_{\left[f_{\varepsilon}\right]_{N, s} \star_{\lambda, s}\left[g_{\varepsilon}\right]_{N, s} \mid}^{s}\right\rangle \alpha\right) \\
& =\frac{1}{\prod_{i=1}^{N+M}\left\|\left(h_{i}\right)_{\varepsilon}\right\|_{1}} \int_{N+M} \mathbb{1}_{\left\{F_{s}>\alpha\right\}}(\bar{w}) \prod_{i=1}^{N+M} \mathbb{1}_{\left[\varepsilon, h_{i}\left(x_{i}\right)\right]}\left(z_{i}\right) d \bar{w} \\
& \quad=\frac{1}{\prod_{i=1}^{N+M}\left\|\left(h_{i}\right)_{\varepsilon}\right\|_{1}} \int_{[0, \infty)^{N+M}}\left(\int_{\left(\mathbb{R}^{n}\right)^{N+M}} \mathbb{1}_{\left\{F_{s}>\alpha\right\}}(\bar{w}) \prod_{i=1}^{N+M} \mathbb{1}_{\left[\varepsilon, h_{i}\left(x_{i}\right)\right]}\left(z_{i}\right) d \bar{x}\right) d \bar{z} \\
& \quad \geq \frac{1}{\prod_{i=1}^{N+M}\left\|\left(h_{i}\right)_{\varepsilon}^{*}\right\|_{1}} \int_{[0, \infty)^{N+M}}\left(\int_{\left(\mathbb{R}^{n}\right)^{N+M}} \mathbb{1}_{\left\{F_{s}>\alpha\right\}}(\bar{w}) \prod_{i=1}^{N+M} \mathbb{1}_{\left[\varepsilon, h_{i}^{*}\left(x_{i}\right)\right]}\left(z_{i}\right) d \bar{x}\right) d \bar{z} \\
& \quad=\frac{1}{\prod_{i=1}^{N+M}\left\|\left(h_{i}\right)_{\varepsilon}^{*}\right\|_{1}} \int_{N+M} \mathbb{1}_{\left\{F_{s}>\alpha\right\}}(\bar{w}) \prod_{i=1}^{N+M} \mathbb{1}_{\left[\varepsilon, h_{i}^{*}\left(x_{i}\right)\right]}\left(z_{i}\right) d \bar{w} \\
& \quad=\mathbb{P}\left(\mid \mathcal{K}_{\left[f_{\varepsilon}^{*}\right]_{N, s} \star_{\lambda, s}\left[g_{\varepsilon}^{*}\right]_{N, s}}>\alpha\right) .
\end{aligned}
$$

The result now follows from (3.23) applied to $f_{\varepsilon}^{*}$ and $g_{\varepsilon}^{*}$. 


\section{Chapter 4}

\section{Stochastic Forms of Brunn's}

\section{Principle}

The results from Chapter 3 open a new path to extend the study of stochastic functional inequalities to operations beyond those of the Prékopa-Leindler inequality. In this part of the dissertation, we obtain a stochastic form of dimensional Brunn's principle, which corresponds to the $s$-concave case, for $s \in(-1 / n, \infty)$.

Due to the limitation of the method used to prove Theorem 1.5, we consider an alternative method, inspired by Rinott, using an epigraph interpretation. We attach an $(n+1)$-dimensional product measure to the epigraphs, finite on the positive rays, that recovers the integral of the function. This alternative technique is connected to shadow systems for epigraphs, used to show Theorem 1.7.

\subsection{Rinott's approach to s-concave functions}

Rinott [80] provides a geometric proof of the inequalities (1.27) by deriving integral inequalities for functions using certain higher-dimensional measures. We start this section by recalling his approach.

For $A \subseteq \mathbb{R}^{n}$, we define the epigraph of $f$ on $A$ in $\mathbb{R}^{n}$ by

$$
E_{A}(f)=\{(x, z) \in A \times \mathbb{R}: f(x) \leq z\}
$$


Analogously we define the hypograph of $f$ on $A$ by

$$
H_{A}(f)=\{(x, z) \in A \times[0, \infty): f(x) \geq z\}
$$

When we omit the subscript $A$, we assume that $A$ is the support of $f$.

Let $f: \mathbb{R}^{n} \rightarrow[0, \infty)$ be an $s$-concave function for $s \in(-1 / n, \infty)$, and $\nu$ a measure on $\mathbb{R}^{n} \times \mathbb{R}$ such that

$$
d \nu\left(x_{1}, \ldots, x_{n+1}\right)=h\left(x_{n+1}\right) d x_{1} \cdots d x_{n+1},
$$

for some continuous function $h: \mathbb{R} \rightarrow \mathbb{R}$. With this setup, we can express the integral of $f$ in terms of the $\nu$-measure of the epigraph or hypograph of a transformation of it. Moreover,

$$
\int_{A} f(x) d x= \begin{cases}\nu\left(E_{A}\left(f^{s}\right)\right), & \text { for } h\left(x_{n+1}\right)=-\frac{1}{s} x_{n+1}^{\frac{1}{s}-1}, \text { if } s<0 . \\ \nu\left(E_{A}(-\log f)\right), & \text { for } h\left(x_{n+1}\right)=e^{-x_{n+1}}, \quad \text { if } s=0 . \\ \nu\left(H_{A}\left(f^{s}\right)\right), & \text { for } h\left(x_{n+1}\right)=\frac{1}{s} x_{n+1}^{\frac{1}{s}-1},\end{cases}
$$

Notice the $s$-concavity of $f$ implies the convexity of $H_{A}\left(f^{s}\right), E_{A}(-\log f)$, and $E_{A}\left(f^{s}\right)$ respectively.

We use the notation in (3.6) for $K, L \subseteq \mathbb{R}^{n}$; similarly for $x_{1}, x_{2} \in \mathbb{R}^{n}$ and $\lambda \in[0,1]$, let $x_{1}+{ }_{\lambda} x_{2}=\lambda x_{1}+(1-\lambda) x_{2}$. For convex functions $\varphi, \psi: \mathbb{R}^{n} \rightarrow \mathbb{R}$ and $\lambda \in[0,1]$, we define their infimal convolution by

$$
\left(\varphi \square_{\lambda} \psi\right)(v)=\inf _{v=x_{1}+\lambda_{\lambda} x_{2}}\left\{\mathcal{M}_{\lambda}^{1}\left(\varphi\left(x_{1}\right), \psi\left(x_{2}\right)\right)\right\}
$$

so that

$$
E\left(\varphi \square_{\lambda} \psi\right)=E(\varphi)+{ }_{\lambda} E(\psi)
$$

Let $f, g: \mathbb{R}^{n} \rightarrow[0, \infty)$ be $s$-concave functions. When $s<0, f^{s}$ and $g^{s}$ are convex 
and

$$
\begin{aligned}
\left(f \star_{\lambda, s} g\right)^{s}(v) & =\left(\sup _{v=x_{1}+{ }_{\lambda} x_{2}} \mathcal{M}_{\lambda}^{1}\left(f^{s}\left(x_{1}\right), g^{s}\left(x_{2}\right)\right)^{1 / s}\right)^{s} \\
& =\inf _{v=x_{1}+{ }_{\lambda} x_{2}} \mathcal{M}_{\lambda}^{1}\left(f^{s}\left(x_{1}\right), g^{s}\left(x_{2}\right)\right) \\
& =\left(f^{s} \square_{\lambda} g^{s}\right)(v),
\end{aligned}
$$

and we have

$$
E\left(\left(f \star_{\lambda, s} g\right)^{s}\right)=E\left(f^{s}\right)+{ }_{\lambda} E\left(g^{s}\right) .
$$

For $s=0,-\log f$ and $-\log g$ are convex and

$$
\begin{aligned}
-\log \left(f \star_{\lambda, 0} g\right)(v) & =-\log \left(\sup _{v=x_{1}+\lambda_{2} x_{2}}\left\{\mathcal{M}_{\lambda}^{0}\left(f\left(x_{1}\right), g\left(x_{2}\right)\right)\right\}\right) \\
& =\inf _{v=x_{1}+\lambda_{2} x_{2}}\left\{-\log \mathcal{M}_{\lambda}^{0}\left(f\left(x_{1}\right), g\left(x_{2}\right)\right)\right\} \\
& =\left((-\log f) \square_{\lambda}(-\log g)\right)(v),
\end{aligned}
$$

which implies

$$
E\left(-\log \left(f \star_{\lambda, 0} g\right)\right)=E(-\log f)+{ }_{\lambda} E(-\log g) .
$$

Lastly, for $s>0,-f^{s},-g^{s}$ are convex and

$$
\begin{aligned}
-\left(f \star_{\lambda, s} g\right)^{s}(v) & =-\sup _{v=x_{1}+{ }_{\lambda} x_{2}} \mathcal{M}_{\lambda}^{1}\left(f^{s}\left(x_{1}\right), g^{s}\left(x_{2}\right)\right) \\
& =\inf _{v=x_{1}+{ }_{\lambda} x_{2}} \mathcal{M}_{\lambda}^{1}\left(-f^{s}\left(x_{1}\right),-g^{s}\left(x_{2}\right)\right) \\
& =\left(-f^{s} \square_{\lambda}-g^{s}\right)(v),
\end{aligned}
$$

from which it follows that

$$
H\left(\left(f \star_{\lambda, s} g\right)^{s}\right)=H\left(f^{s}\right)+{ }_{\lambda} H\left(g^{s}\right) .
$$




\section{$4.2 \mathcal{M}$-addition and multiple integral rearrange- ment inequalities}

To connect with the epigraphs and hypographs from $\S 4$.1, we use $\mathcal{M}$-combinations of rays and line segments in $\mathbb{R}^{n+1}$. Let $C \subseteq \mathbb{R}^{N}$ be a compact, convex set contained in the positive orthant, $\rho_{1}, \ldots, \rho_{N} \in \mathbb{R}$, and $x_{1}, \ldots, x_{N} \in \mathbb{R}^{n}$. We define the rays

$$
R_{\rho_{i}}\left(x_{i}\right)=\left\{\left(x_{i}, r\right) \in \mathbb{R}^{n} \times \mathbb{R}: \rho_{i} \leq r\right\}
$$

and the line segments

$$
\widetilde{R}_{\rho_{i}}\left(x_{i}\right)=\left\{\left(x_{i}, r\right) \in \mathbb{R}^{n} \times[0, \infty): \rho_{i} \geq r\right\}
$$

Accordingly,

$$
\oplus_{C}\left(R_{\rho_{1}}\left(x_{1}\right), \ldots, R_{\rho_{N}}\left(x_{N}\right)\right) \quad \text { and } \quad \oplus_{C}\left(\widetilde{R}_{\rho_{1}}\left(x_{1}\right), \ldots, \widetilde{R}_{\rho_{N}}\left(x_{N}\right)\right)
$$

form the epigraph of a convex function and the hypograph of a concave function, respectively. By choosing $C=\operatorname{conv}\left\{e_{1}, \ldots, e_{N}\right\}$, one simply takes the convex hull of the rays or line segments, respectively. We now show that Theorem 3.1 interfaces well with our approach.

Proposition 4.1. Let $\rho_{1}, \ldots, \rho_{N} \in \mathbb{R}$ and $C$ a compact convex set contained in the positive orthant. Then the function $F:\left(\mathbb{R}^{n}\right)^{N} \rightarrow[0, \infty)$ defined by

$$
F\left(x_{1}, \ldots, x_{N}\right)=\nu\left(\oplus_{C}\left(R_{\rho_{1}}\left(x_{1}\right), \ldots, R_{\rho_{N}}\left(x_{N}\right)\right)\right)
$$

is Steiner convex.

Proof. Let $\theta \in \mathbb{S}^{n-1}$ and $y_{1}, \ldots, y_{N} \in \theta^{\perp}$. Let $\left(s_{1}, \ldots, s_{N}\right),\left(t_{1}, \ldots, t_{N}\right) \in \mathbb{R}^{N}$ and 
$\tau \in(0,1)$. For $i=1, \ldots, N$, write

$$
y_{i}+\left(\tau s_{i}+(1-\tau) t_{i}\right) \theta=\left(y_{i}+t_{i} \theta\right)+\tau\left(t_{i}-s_{i}\right) \theta
$$

and apply Proposition 4.3 with $x_{i}=y_{i}+t_{i} \theta, \lambda_{i}=t_{i}-s_{i}$ and $t=\tau$ to obtain the convexity in $\tau$. Lastly, the sets

$$
\oplus_{C}\left(R_{\rho_{1}}\left(y_{1}+t_{1} \theta\right), \ldots, R_{\rho_{N}}\left(y_{N}+t_{N} \theta\right)\right)
$$

and

$$
\oplus_{C}\left(R_{\rho_{1}}\left(y_{1}-t_{1} \theta\right), \ldots, R_{\rho_{N}}\left(y_{N}-t_{N} \theta\right)\right)
$$

are reflections of one another and so the evenness condition required for Steiner convexity holds.

Next, we state the analogous proposition involving the line segments (4.6b); the proof follows the same line.

Proposition 4.2. Let $\rho_{1}, \ldots, \rho_{N} \in \mathbb{R}$ and $C$ a compact convex set contained in the positive orthant. Then the function $F:\left(\mathbb{R}^{n}\right)^{N} \rightarrow[0, \infty)$ defined by

$$
F\left(x_{1}, \ldots, x_{N}\right)=\nu\left(\oplus_{C}\left(\widetilde{R}_{\rho_{1}}\left(x_{1}\right), \ldots, \widetilde{R}_{\rho_{N}}\left(x_{N}\right)\right)\right)
$$

is Steiner convex.

\subsection{Shadow systems of $s$-concave functions}

In this section, we recall the notion of linear parameter and shadow systems of convex sets and extend these to $s$-concave functions. We establish a corresponding convexity property in the functional setting.

Recall the notation for linear parameter systems from the introduction: for an index set $I$, bounded sets $\left\{x_{i}\right\}_{i \in I} \subseteq \mathbb{R}^{n}$ and $\left\{\lambda_{i}\right\}_{i \in I} \subseteq \mathbb{R}$ and $\theta \in \mathbb{S}^{n-1}, a \leq t \leq b$, 
we set

$$
K_{t}=\operatorname{conv}\left\{x_{i}+\lambda_{i} t \theta: i \in I\right\}, \quad t \in[a, b]
$$

In the notation of $\S 2.1$, we use $u\left(K_{t}, \cdot\right), \ell\left(K_{t}, \cdot\right), P=P_{\theta^{\perp}}$, and we set $D=P K$. Define $L(x)=u\left(K_{t}, x\right)-\ell\left(K_{t}, x\right)$. Rogers and Shephard proved the fundamental fact that for $x \in D, t \mapsto L\left(K_{t}, x\right)$ is convex. Consequently, the map

$$
t \mapsto\left|K_{t}\right|=\int_{D} L\left(K_{t}, x\right) d x
$$

is a convex function of $t$. For background on shadow systems, see e.g., [22, 63, 87], and $[88, \S 10.4]$.

We use certain linear parameter systems for a finite index set $I=\{1, \ldots, N\}$ associated to the $\oplus_{C}$ operations of the previous section for epigraphs and hypographs.

Proposition 4.3. Let $C$ be a compact convex set in $\mathbb{R}^{N}$ contained in the positive orthant. Let $\left\{x_{i}\right\}_{i=1}^{N} \subseteq \mathbb{R}^{n},\left\{z_{i}\right\} \subseteq(0, \infty)$ and $\left\{\lambda_{i}\right\}_{i=1}^{N} \subseteq \mathbb{R}$. For $s \neq 0$, let $\rho_{i}(s)=z_{i}^{s}$ and for $s=0$ let $\rho_{i}(s)=-\log z_{i}$. For $a<b$ and $t \in[a, b]$, let

$$
E_{t}^{s}=\oplus_{C}\left(\left\{R_{\rho_{i}(s)}\left(x_{i}+\lambda_{i} t \theta\right)\right\}_{i=1}^{N}\right) \quad(s \geq 0)
$$

and

$$
H_{t}^{s}=\oplus_{C}\left(\left\{\widetilde{R}_{\rho_{i}(s)}\left(x_{i}+\lambda_{i} t \theta\right)\right\}_{i=1}^{N}\right) \quad(s<0) .
$$

Then for $\nu$ as in (4.2), $t \mapsto \nu\left(E_{t}^{s}\right)$ and $t \mapsto \nu\left(H_{t}^{s}\right)$ are convex.

Proof. Let $s \geq 0$. For $z \in \mathbb{R}$, write $\pi_{z}=e_{n+1}^{\perp}+z e_{n+1}$ so that

$$
\nu\left(E_{t}^{s}\right)=\int_{\mathbb{R}}\left|E_{t}^{s} \cap \pi_{z}\right| h(z) d z
$$

Thus it suffices to show that for fixed $z, t \mapsto\left|E_{t}^{s} \cap \pi_{z}\right|$ is convex. We have

$$
E_{t}^{s}=\left\{\sum_{i=1}^{N} c_{i}\left(x_{i}+r_{i} e_{n+1}\right)+\left(\sum_{i=1}^{N} c_{i} \lambda_{i}\right) t \theta: c \in C, \rho_{i}(s) \leq r_{i}\right\}
$$


As noted in the previous section, since $C$ is compact, convex and contained in the positive orthant, $E_{t}^{s}$ is convex. For $c \in C$, we write $x_{c}=\sum_{i=1}^{N} c_{i} x_{i}, r_{c}=\sum_{i=1}^{N} c_{i} r_{i}$, and $\lambda_{c}=\sum_{i=1}^{N} c_{i} \lambda_{i}$. For $x_{c}+r_{c} e_{n+1}+\lambda_{c} t \theta \in E_{t}^{s} \cap \pi_{z}$, we have $r_{c}=z$ and the sets $\left\{x_{c}+z e_{n+1}\right\},\left\{\lambda_{c}\right\}$ are bounded. Thus $E_{t}^{s} \cap \pi_{z}$ is a linear parameter system of convex sets indexed by $C$. Then $t \mapsto\left|E_{t}^{s} \cap \pi_{z}\right|$ is convex by (4.9). The argument for $s<0$ is analogous.

Next, we define a linear parameter system of $s$-concave functions. Let $I$ be an index set, and $\left\{\left(x_{i}, z_{i}\right)\right\}_{i \in I} \subseteq \mathbb{R}^{n} \times[0, \infty)$ a collection of points under the graph of some integrable $s$-concave function. For $s \geq 0$, let $T_{\left\{w_{i}\right\}, s}$ and be the least $s$-concave function above $\left\{w_{i}\right\}$. For $s<0$, let $T_{\left\{w_{i}\right\}, s}$ be the greatest $s$-concave function beneath $\left\{w_{i}\right\}$. More explicitly, $T_{\left\{w_{i}\right\}, s}$ is supported on $\operatorname{conv}\left\{x_{i}: i \in I\right\}$ and given by

$$
T_{\left\{w_{i}\right\}, s}(x)= \begin{cases}\inf \left\{z^{1 / s}:(x, z) \in \mathcal{P}_{\left\{w_{i}\right\}}\right\}, & \text { if } s<0 \\ \sup \left\{e^{z}:(x, z) \in \mathcal{P}_{\left\{w_{i}\right\}}\right\}, & \text { if } s=0, \\ \sup \left\{z^{1 / s}:(x, z) \in \mathcal{P}_{\left\{w_{i}\right\}}\right\}, & \text { if } s>0,\end{cases}
$$

where

$$
\mathcal{P}_{\left\{w_{i}\right\}}= \begin{cases}\operatorname{conv}\left\{\left(x_{i}, z_{i}^{s}\right)\right\}_{i \in I}, & \text { if } s \neq 0 . \\ \operatorname{conv}\left\{\left(x_{i}, \log z_{i}\right)\right\}_{i \in I}, & \text { if } s=0 .\end{cases}
$$

With the above notation, we assume that $w_{i}(t)=\left(x_{i}+\lambda_{i} t \theta, z_{i}\right), a \leq t \leq b$. Then setting $f_{t, s}=T_{\left\{w_{i}(t)\right\}, s}$, we call the family $\left\{f_{t, s}\right\}$ a linear parameter system of $s$-concave functions. The convexity property corresponding to (4.9) reads as follows.

Proposition 4.4. Let $\left\{f_{t, s}\right\}$ be a linear parameter system of s-concave functions. Then

$$
t \mapsto \int_{\mathbb{R}^{n}} f_{t}(v) d v
$$


is a convex function.

Proof. As for linear parameter systems of convex sets, we can assume without loss generality that $I$ is finite, say $I=\{1, \ldots, N\}$. We take $C$ to be $\operatorname{conv}\left\{e_{1}, \ldots, e_{N}\right\}$. In the notation of the previous proposition, we have

$$
E_{t}^{s}=\operatorname{conv}\left\{R_{\rho_{i}(s)}\left(x_{i}+\lambda_{i} t \theta\right)\right\}_{i=1}^{N}
$$

and $E_{t}^{s}=E\left(-\log f_{t, s}\right)$ for $s=0$, while $E_{t}^{s}=E\left(f_{t, s}^{s}\right)$ for $s<0$. Similarly,

$$
H_{t}^{s}=\operatorname{conv}\left\{\widetilde{R}_{\rho_{i}(s)}\left(x_{i}+\lambda_{i} t \theta\right)\right\}_{i=1}^{N},
$$

hence $H_{t}^{s}=H\left(f_{t, s}^{s}\right)$ for $s>0$. By (4.2), we have

$$
\int f_{t, s}(v) d v= \begin{cases}\nu\left(E_{t}^{s}\right), & \text { for } h\left(x_{n+1}\right)=-\frac{1}{s} x_{n+1}^{\frac{1}{s}-1}, \text { if } s<0 . \\ \nu\left(E_{t}^{s}\right), & \text { for } h\left(x_{n+1}\right)=e^{-x_{n+1},}\end{cases}
$$

and we can conclude the proof by applying Proposition 4.3.

Shephard [89] introduced shadow systems to extend linear parameter systems. Given a convex body $K \subseteq \mathbb{R}^{n}$, a bounded function $\alpha: K \rightarrow \mathbb{R}$ and $t \in[a, b]$, a shadow system in direction $\theta \in \mathbb{S}^{n-1}$ is a family of convex sets

$$
K_{t}=\operatorname{conv}\{x+\alpha(x) t \theta: x \in K\}
$$

Then $K_{t}=P_{t} \bar{K}$, where $\bar{K}=\{(x, \alpha(x)): x \in K\}$ and $P_{t}: \mathbb{R}^{n} \times \mathbb{R} \rightarrow \mathbb{R}^{n}$ is the projection parallel to $e_{n+1}-t \theta$ given by $P_{t}(x, y)=x+t y \theta$. Conversely, for any convex body $\bar{K} \subseteq \mathbb{R}^{n+1}, \theta \in \mathbb{S}^{n-1}$, and $t \in[a, b]$, the family $\left\{K_{t}\right\}=\left\{P_{t} \bar{K}\right\} \subseteq \mathbb{R}^{n}$ is a shadow system of convex bodies.

The correspondence between linear parameter systems of $s$-concave functions 
and epigraphs/hypographs in the proof of Proposition 4.4 affords a similar extension to shadow systems. We can simply start with shadow systems of epigraphs or hypographs. Let $\mathcal{E} \subseteq \mathbb{R}^{n+2}$ be the epigraph of a convex function $\Phi: \mathbb{R}^{n+1} \rightarrow$ $[0, \infty), \theta \in \mathbb{S}^{n-1}, t \in[a, b]$, and the projection $P_{t}$ from $\mathbb{R}^{n} \times \mathbb{R} \times \mathbb{R}$ onto $\mathbb{R}^{n} \times \mathbb{R}$ parallel to $e_{n+2}-t \theta$ given by

$$
P_{t}(x, z, y)=(x+t y \theta, z)
$$

Then the family $\left\{E_{t}\right\}=\left\{P_{t} \mathcal{E}\right\}$ is a shadow system of epigraphs of convex functions, where

$$
E_{t}=\operatorname{conv}\left\{(x+y t \theta, z) \in \mathbb{R}^{n} \times \mathbb{R}:(x, y, z) \in \mathcal{E}\right\} .
$$

Consequently, let $\phi: \mathbb{R}^{n} \rightarrow[0, \infty)$ be a convex function, $\alpha: E(\phi) \rightarrow \mathbb{R}$ a function such that $\left.\alpha\right|_{E(\phi) \cap \pi_{z}}: \mathbb{R}^{n} \rightarrow \mathbb{R}$ is bounded for all $z \in \mathbb{R}$. Consider $\Phi: \mathbb{R}^{n+1} \rightarrow \mathbb{R}$ described by its epigraph $E(\Phi)=\operatorname{conv}\{(x, z, \alpha(x, z)): \phi(x) \leq z\}$ and set

$$
(E(\phi))_{t}=P_{t} E(\Phi)=\operatorname{conv}\{(x+\alpha(x, z) t \theta, z): \phi(x) \leq z\}
$$

Then we define the shadow system of convex functions $\phi_{t}: \mathbb{R}^{n} \rightarrow[0, \infty)$ in direction $\theta \in \mathbb{S}^{n}$ by

$$
\phi_{t}(x)=\inf \left\{z:(x, z) \in(E(\phi))_{t}\right\}
$$

Analogously, given the hypograph $\mathcal{H} \subseteq \mathbb{R}^{n+2}$ of a concave function $\Psi: \mathbb{R}^{n+1} \rightarrow$ $[0, \infty)$ the family $\left\{H_{t}\right\}=\left\{P_{t} \mathcal{H}\right\}$ is a shadow system of hypographs of concave functions where

$$
H_{t}=\operatorname{conv}\left\{(x+y t \theta, z) \in \mathbb{R}^{n} \times \mathbb{R}:(x, z, y) \in \mathcal{H}\right\}
$$

Similarly, for a concave function $\psi: \mathbb{R}^{n} \rightarrow[0, \infty), \alpha: H(\psi) \rightarrow \mathbb{R}$ a function such that $\left.\alpha\right|_{H(\psi) \cap \pi_{z}}: \mathbb{R}^{n} \rightarrow \mathbb{R}$ is bounded for all $z \in \mathbb{R}$. Consider the function 
$\Psi: \mathbb{R}^{n+1} \rightarrow \mathbb{R}$ with hypograph $H(\Psi)=\{(x, z, \alpha(x, z)): 0 \leq z \leq \psi(x)\}$ and set

$$
(H(\psi))_{t}=P_{t} H(\Psi)=\operatorname{conv}\{(x+\alpha(x, z) t \theta, z): 0 \leq z \leq \psi(x)\}
$$

Then we define the shadow system of concave functions $\psi_{t}: \mathbb{R}^{n} \rightarrow \mathbb{R}$ by

$$
\psi_{t}(x)=\sup \left\{z:(x, z) \in(H(\psi))_{t}\right\}
$$

Proposition 4.5. For shadow systems of epigraphs $E_{t}$ and hypographs $H_{t}$ and $\nu$ as in (4.2), we have that $t \mapsto \nu\left(E_{t}\right)$ and $t \mapsto \nu\left(H_{t}\right)$ are convex.

Proof. Let $\nu$ be as in (4.2). Then

$$
\nu\left(E_{t}\right)=\int_{\mathbb{R}}\left|E_{t} \cap \pi_{z}\right| h(z) d z
$$

For each $z$, the restriction of the epigraph $E_{t}$ to the parallel hyperplane $\pi_{z}$ is a shadow systems of convex bodies. Thus the convexity of $t \mapsto \nu\left(E_{t}\right)$ follows from the convexity of the function (4.9). The proof for $H_{t}$ is analogous.

\subsection{Random epigraphs and hypographs}

In this section, we take our stochastic model for $[f]_{N}$, as defined in the introduction, and reformulate it in terms of epigraphs and hypographs. Thus for an integrable $s$-concave function $f: \mathbb{R}^{n} \rightarrow[0, \infty)$, we sample independent random vectors $\left(X_{1}, Z_{1}\right), \ldots,\left(X_{N}, Z_{N}\right)$ according to Lebesgue measure on 1.22. For $C_{N}=\operatorname{conv}\left\{e_{1}, \ldots, e_{N}\right\}$, we set

$$
\begin{aligned}
& {\left[E^{s}\right]_{N}= \begin{cases}\oplus_{C_{N}}\left(R_{Z_{1}^{s}}\left(X_{1}\right), \ldots, R_{Z_{N}^{s}}\left(X_{N}\right)\right), & \text { if } s<0, \\
\oplus_{C_{N}}\left(R_{-\log Z_{1}}\left(X_{1}\right), \ldots, R_{-\log Z_{N}}\left(X_{N}\right)\right), \text { if } s=0 .\end{cases} } \\
& {\left[H^{s}\right]_{N}=\oplus_{C_{N}}\left(\widetilde{R}_{Z_{1}^{s}}\left(X_{1}\right), \ldots, \widetilde{R}_{Z_{N}^{s}}\left(X_{N}\right)\right), \quad \text { if } s>0 .}
\end{aligned}
$$


With this notation,

$$
\begin{array}{ll}
{\left[E^{s}\right]_{N}=E\left([f]_{N}^{s}\right),} & \text { if } s<0 . \\
{\left[E^{s}\right]_{N}=E\left(-\log [f]_{N}\right),} & \text { if } s=0 . \\
{\left[H^{s}\right]_{N}=H\left([f]_{N}^{s}\right),} & \text { if } s>0
\end{array}
$$

and, by (4.2),

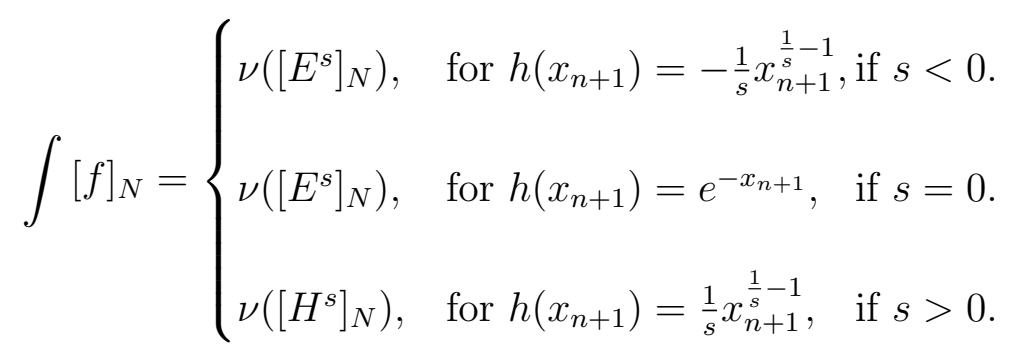

\subsection{Proof of Stochastic Brunn's principle}

Proof of Theorem 1.7. Let $w_{i}=\left(x_{i}, z_{i}\right) \in \mathbb{R}^{n} \times \mathbb{R}$ for $i=1, \ldots, M+N$. For $s \geq 0$, let $T_{\left\{w_{i}\right\}, s}^{(N)}$ and $T_{\left\{w_{i}\right\}, s}^{(M)}$ be the least $s$-concave functions above the collections $\left\{w_{i}\right\}_{i \leq N}$ and $\left\{w_{i}\right\}_{N+1 \leq i \leq M}$, respectively; similarly, when $s<0$, let $T_{\left\{w_{i}\right\}}^{(N)}$ and $T_{\left\{w_{i}\right\}}^{(M)}$ be the greatest $s$-concave functions beneath the respective collections $\left\{w_{i}\right\}_{i \leq N}$ and $\left\{w_{i}\right\}_{N+1 \leq i \leq M}$. With this notation, we set

$$
F\left(w_{1}, \ldots, w_{N+M}\right)=\int_{\mathbb{R}^{n}} T_{\left\{w_{i}\right\}}^{(N)} \star_{\lambda} T_{\left\{w_{i}\right\}}^{(M)}(v) d v .
$$

Let $f, g: \mathbb{R}^{n} \rightarrow[0, \infty)$ be integrable $s$-concave functions for $s \in(-1 / n, \infty)$. Sample independent random vectors $W_{i}=\left(X_{i}, Z_{i}\right), i=1, \ldots, N+M$ uniformly according to the Lebesgue measure on $G_{f}$ for $i=1, \ldots, N$ and $G_{g}$ for $i=N+$ 
$1, \ldots, N+M$. Then the random functions $[f]_{N},[g]_{M}$ satisfy

$$
\begin{aligned}
& \mathbb{P}\left(\int_{\mathbb{R}^{n}}[f]_{N} \star_{\lambda, s}[g]_{M}(v) d v>\alpha\right) \\
& \quad=\frac{1}{\prod_{i=1}^{M+N}\left\|k_{i}\right\|_{1}} \int_{N+M} \mathbb{1}_{\{F>\alpha\}}(\bar{w}) \prod_{i=1}^{N+M} \mathbb{1}_{\left[0, k_{i}\left(x_{i}\right)\right]}\left(z_{i}\right) d \bar{w}
\end{aligned}
$$

where $\int_{N+M}$ is the integral on $\left(\mathbb{R}^{n} \times[0, \infty)\right)^{N+M}, k_{i}=f$ for $i=1, \ldots, N, k_{i}=g$ for $i=N+1, \ldots, N+M$, and we use the same notation as in 3.18. Also we write $C_{N}=\operatorname{conv}\left\{e_{1}, \ldots, e_{N}\right\}$ and $\widehat{C}_{M}=\operatorname{conv}\left\{e_{N+1}, \ldots, e_{N+M}\right\}$, and consider $\nu$ as in (4.2) for each case.

Case s > 0: By (4.17b) and (4.5) it follows

$$
\begin{aligned}
H\left(\left([f]_{N} \star_{\lambda, s}[g]_{M}\right)^{s}\right) & =\oplus_{C_{N}}\left(\left\{\widetilde{R}_{Z_{i}^{s}}\left(X_{i}\right)\right\}_{i=1}^{N}\right)+_{\lambda} \oplus_{\widehat{C}_{M}}\left(\left\{\widetilde{R}_{Z_{i}^{s}}\left(X_{i}\right)\right\}_{i=N+1}^{N+M}\right) \\
& =\oplus_{C_{N}+\lambda} \widehat{C}_{M}\left(\left\{\widetilde{R}_{Z_{i}^{s}}\left(X_{i}\right)\right\}_{i=1}^{N+M}\right),
\end{aligned}
$$

hence

$$
\int_{\mathbb{R}^{n}}[f]_{N} \star_{\lambda, s}[g]_{M}(v) d v=\nu\left(\oplus_{C_{N}+\lambda_{\lambda} \widehat{C}_{M}}\left(\left\{\widetilde{R}_{Z_{i}^{s}}\left(X_{i}\right)\right\}_{i=1}^{N+M}\right)\right) .
$$

By (4.19), Fubini, Proposition 4.1, and Theorem 3.1, we have

$$
\begin{aligned}
& \mathbb{P}\left(\int_{\mathbb{R}^{n}}[f]_{N} \star_{\lambda, s}[g]_{M}(v) d v>\alpha\right) \\
& \quad=\frac{1}{\prod_{i=1}^{M+N}\left\|k_{i}\right\|_{1}^{N+M}} \int_{\left(\mathbb{R}^{n} \times[0, \infty)\right)^{N+M}} \mathbb{1}_{\{F>\alpha\}}(\bar{w}) \prod_{i=1}^{N+M} \mathbb{1}_{\left[0, k_{i}\left(x_{i}\right)\right]}\left(z_{i}\right) d \bar{w} \\
& \quad=\frac{1}{\prod_{i=1}^{M+N}\left\|k_{i}\right\|_{1}^{N+M}} \int_{[0, \infty)^{N+M}}\left(\int_{\left(\mathbb{R}^{n}\right)^{N+M}} \mathbb{1}_{\{F>\alpha\}}(\bar{w}) \prod_{i=1}^{N+M} \mathbb{1}_{\left[0, k_{i}\left(x_{i}\right)\right]}\left(z_{i}\right) d \bar{x}\right) d \bar{z} \\
& \quad \geq \frac{1}{\prod_{i=1}^{M+N}\left\|k_{i}^{*}\right\|_{1}^{N+M}} \int_{[0, \infty)^{N+M}}\left(\int_{\left(\mathbb{R}^{n}\right)^{N+M}} \mathbb{1}_{\{F>\alpha\}}(\bar{w}) \prod_{i=1}^{N+M} \mathbb{1}_{\left[0, k_{i}^{*}\left(x_{i}\right)\right]}\left(z_{i}\right) d \bar{x}\right) d \bar{z} \\
& \quad=\frac{1}{\prod_{i=1}^{M+N}\left\|k_{i}^{*}\right\|_{1}^{N+M}} \int_{\left(\mathbb{R}^{n} \times[0, \infty)\right)^{N}} \mathbb{1}_{\{F>\alpha\}}(\bar{w}) \prod_{i=1}^{N+M} \mathbb{1}_{\left[0, k_{i}^{*}\left(x_{i}\right)\right]}\left(z_{i}\right) d \bar{w} \\
& \quad=\mathbb{P}\left(\int_{\mathbb{R}^{n}}\left[f^{*}\right]_{N} \star_{\lambda, s}\left[g^{*}\right]_{M}(v) d v>\alpha\right) .
\end{aligned}
$$


Case $\mathbf{s}=\mathbf{0}$ : Using (4.17a), (4.4), we have

$$
E\left(-\log \left([f]_{N} \star_{\lambda, 0}[g]_{M}\right)\right)=\oplus_{C_{N}{ }_{\lambda} \widehat{C}_{M}}\left(\left\{R_{-\log Z_{i}}\left(X_{i}\right)\right\}_{i=1}^{N+M}\right),
$$

SO

$$
\int_{\mathbb{R}^{n}}[f]_{N} \star_{\lambda, 0}[g]_{M}(v) d v=\nu\left(\oplus_{C_{N}+{ }_{\lambda} \widehat{C}_{M}}\left(\left\{R_{-\log Z_{i}}\left(X_{i}\right)\right\}_{i=1}^{N+M}\right)\right) .
$$

It follows as before

$$
\mathbb{P}\left(\int_{\mathbb{R}^{n}}[f]_{N} \star_{\lambda, 0}[g]_{M}(v) d v>\alpha\right) \geq \mathbb{P}\left(\int_{\mathbb{R}^{n}}\left[f^{*}\right]_{N} \star_{\lambda, 0}\left[g^{*}\right]_{M}(v) d v>\alpha\right)
$$

Case $\mathbf{s}<$ 0: It follows by (4.17a) and (4.4) that

$$
E\left(\left([f]_{N} \star_{\lambda, s}[g]_{M}\right)^{s}\right)=\oplus_{C_{N}+\lambda \widehat{C}_{M}}\left(\left\{R_{Z_{i}^{s}}\left(X_{i}\right)\right\}_{i=1}^{N+M}\right),
$$

hence

$$
\int_{\mathbb{R}^{n}}[f]_{N} \star_{\lambda, s}[g]_{M}(v) d v=\nu\left(\oplus_{C_{N}+{ }_{\lambda} \widehat{C}_{M}}\left(\left\{R_{Z_{i}^{s}}\left(X_{i}\right)\right\}_{i=1}^{N+M}\right)\right) .
$$

It follows as before

$$
\mathbb{P}\left(\int_{\mathbb{R}^{n}}[f]_{N} \star_{\lambda, s}[g]_{M}(v) d v>\alpha\right) \geq \mathbb{P}\left(\int_{\mathbb{R}^{n}}\left[f^{*}\right]_{N} \star_{\lambda, s}\left[g^{*}\right]_{M}(v) d v>\alpha\right) .
$$




\section{BIBLIOGRAPHY}

[1] M. Alexander, M. Fradelizi, and A. Zvavitch. Polytopes of maximal volume product. Discrete $\&$ Computational Geometry, 62(3):583-600, 2019.

[2] S. Artstein-Avidan, D. I. Florentin, and A. Segal. Functional BrunnMinkowski inequalities induced by polarity. Advances in Mathematics, 364:107006, 2020.

[3] S. Artstein-Avidan, A. Giannopoulos, and V. D. Milman. Asymptotic geometric analysis. Part I, volume 202 of Mathematical Surveys and Monographs. American Mathematical Society, Providence, RI, 2015.

[4] S. Artstein-Avidan, B. Klartag, and V. Milman. The Santaló point of a function, and a functional form of the Santaló inequality. Mathematika, 51(12):33-48 (2005), 2004.

[5] S Artstein-Avidan, B Klartag, C Schütt, and E Werner. Functional affineisoperimetry and an inverse logarithmic Sobolev inequality. Journal of Functional Analysis, 262(9):4181-4204, 2012.

[6] K. Ball. Isoperimetric problems in $\ell_{p}$ and sections of convex sets. $\mathrm{PhD}$ thesis, Cambridge, 1986.

[7] F. Barthe and M. Fradelizi. The volume product of convex bodies with many hyperplane symmetries. American Journal of Mathematics, 135(2):311-347, 2013. 
[8] T. Bisztriczky and K. Böröczky. About the centroid body and the ellipsoid of inertia. Mathematika, 48(1-2):1-13, 2001.

[9] W. Blaschke. Über affine Geometrie XI: Lösung des "Vierpunktproblems" von Sylvester aus der Theorie der geometrischen Wahrscheinlichkeiten. Leipziger Berichte, 69:436-453, 1917.

[10] W Blaschke. Vorlesungen über Differentialgeometrie, Band II: Affine Differentialgeometrie, bearb. v. K. Reidemeister. Berlin, 1923.

[11] S. G. Bobkov, A. Colesanti, and I. Fragalà. Quermassintegrals of quasiconcave functions and generalized Prékopa-Leindler inequalities. Manuscripta Math., 143(1-2):131-169, 2014.

[12] C. Borell. Convex set functions in $d$-space. Period. Math. Hungar., 6(2):111$136,1975$.

[13] J. Bourgain and V. Milman. New volume ratio properties for convex symmetric bodies in $\mathbb{R}^{n}$. Inventiones mathematicae, 88(2):319-340, 1987.

[14] H. J. Brascamp and E. H. Lieb. Best constants in Young's inequality, its converse, and its generalization to more than three functions. Advances in Math., 20(2):151-173, 1976.

[15] H. J. Brascamp and E. H. Lieb. On extensions of the Brunn-Minkowski and Prékopa-Leindler theorems, including inequalities for log concave functions, and with an application to the diffusion equation. J. Functional Analysis, 22(4):366-389, 1976.

[16] H. J. Brascamp, E. H. Lieb, and J.M. Luttinger. A general rearrangement inequality for multiple integrals. Journal of functional analysis, 17(2):227237,1974

[17] A. Burchard. A short course on rearrangement inequalities. Avaible at http: //www.math.utoronto.ca/almut/rearrange/pdf. 
[18] H. Busemann. Volume in terms of concurrent cross-sections. Pacific J. Math., $3: 1-12,1953$.

[19] H. Busemann and E. G. Straus. Area and normality. Pacific Journal of Mathematics, 10(1):35-72, 1960.

[20] U Caglar, M Fradelizi, O Guédon, J Lehec, C Schütt, and E Werner. Functional versions of lp-affine surface area and entropy inequalities. International Mathematics Research Notices, 2016(4):1223-1250, 2016.

[21] S. Campi, A. Colesanti, and P. Gronchi. A note on Sylvester's problem for random polytopes in a convex body. Rend. Istit. Mat. Univ. Trieste, 31(12):79-94, 1999.

[22] S. Campi and P. Gronchi. On volume product inequalities for convex sets. Proc. Amer. Math. Soc., 134(8):2393-2402, 2006.

[23] S. Campi and P. Gronchi. Volume inequalities for sets associated with convex bodies. In Integral geometry and convexity, pages 1-15. World Scientific, 2006.

[24] M. Christ. Estimates for the $k$-plane transform. Indiana Univ. Math. J., 33(6):891-910, 1984.

[25] D. Cordero-Erausquin. On matrix-valued log-concavity and related Prékopa and Brascamp-Lieb inequalities. Adv. Math., 351:96-116, 2019.

[26] D. Cordero-Erausquin, M. Fradelizi, G. Paouris, and P. Pivovarov. Volume of the polar of random sets and shadow systems. Math. Ann., 362(3-4):1305$1325,2015$.

[27] D Cordero-Erausquin and B Klartag. Interpolations, convexity and geometric inequalities. In Geometric aspects of functional analysis, pages 151-168. Springer, 2012. 
[28] D. Cordero-Erausquin and B. Maurey. Some extensions of the PrékopaLeindler inequality using Borell's stochastic approach. Studia Math., 238(3):201-233, 2017.

[29] D. Cordero-Erausquin, R. J. McCann, and M. Schmuckenschläger. A Riemannian interpolation inequality à la Borell, Brascamp and Lieb. Invent. Math., 146(2):219-257, 2001.

[30] L. Dalla and D. G. Larman. Volumes of a random polytope in a convex set. In Applied geometry and discrete mathematics, pages 175-180, 1990.

[31] M. Fradelizi, A. Hubard, M. Meyer, E. Roldán-Pensado, and A. Zvavitch. Equipartitions and Mahler volumes of symmetric convex bodies. arXiv preprint arXiv:1904.10765, 2019.

[32] M. Fradelizi and M. Meyer. Some functional forms of Blaschke-Santaló inequality. Math. Z., 256(2):379-395, 2007.

[33] M. Fradelizi and M. Meyer. Some functional inverse Santaló inequalities. Advances in Mathematics, 218(5):1430-1452, 2008.

[34] M. Fradelizi, M. Meyer, and A. Zvavitch. An application of shadow systems to Mahler's conjecture. Discrete $\&$ Computational Geometry, 48(3):721-734, 2012

[35] R. J. Gardner. The Brunn-Minkowski inequality. Bull. Amer. Math. Soc. (N.S.), 39(3):355-405, 2002.

[36] R. J. Gardner, D. Hug, and W. Weil. Operations between sets in geometry. J. Eur. Math. Soc. (JEMS), 15(6):2297-2352, 2013.

[37] R. J. Gardner, D. Hug, and W. Weil. The Orlicz-Brunn-Minkowski theory: a general framework, additions, and inequalities. J. of Differential Geom, 97(3):427-476, 2014. 
[38] A. Giannopoulos, G. Paouris, and B. H. Vritsiou. The isotropic position and the reverse santaló inequality. Israel Journal of Mathematics, 203(1):1-22, 2014 .

[39] A Giannopoulos and A Tsolomitis. Volume radius of a random polytope in a convex body. In Mathematical Proceedings of the Cambridge Philosophical Society, volume 134, pages 13-21. [Cambridge, Eng.] Cambridge Philosophical Society., 2003.

[40] H Groemer. On some mean values associated with a randomly selected simplex in a convex set. Pacific Journal of Mathematics, 45(2):525-533, 1973.

[41] H. Groemer. On the mean value of the volume of a random polytope in a convex set. Arch. Math. (Basel), 25:86-90, 1974.

[42] R. Henstock and A.M. Macbeath. On the measure of sum-sets.(i) the theorems of Brunn, Minkowski, and Lusternik. Proceedings of the London Mathematical Society, 3(1):182-194, 1953.

[43] H. Iriyeh and M. Shibata. Symmetric Mahler's conjecture for the volume product in the three dimensional case. arXiv preprint arXiv:1706.01749, 2017.

[44] R. Karasev. Mahler's conjecture for some hyperplane sections. arXiv preprint arXiv:1902.08971, 2019.

[45] J. Kim and S. Reisner. Local minimality of the volume-product at the simplex. Mathematika, 57(1):121-134, 2011.

[46] B. Klartag. Marginals of geometric inequalities. In Geometric aspects of functional analysis, volume 1910 of Lecture Notes in Math., pages 133-166. Springer, Berlin, 2007.

[47] B. Klartag. Isotropic constants and Mahler volumes. Advances in Mathematics, 330:74-108, 2018. 
[48] B. Klartag and V. D. Milman. Geometry of log-concave functions and measures. Geom. Dedicata, 112:169-182, 2005.

[49] G. Kuperberg. From the Mahler conjecture to Gauss linking integrals. Geometric And Functional Analysis, 18(3):870-892, 2008.

[50] J. Lehec. A direct proof of the functional Santaló inequality. C. R. Math. Acad. Sci. Paris, 347(1-2):55-58, 2009.

[51] L. Leindler. On a certain converse of Hölder's inequality. II. Acta Sci. Math. (Szeged), 33(3-4):217-223, 1972.

[52] E. H. Lieb and M. Loss. Analysis, volume 14 of Graduate Studies in Mathematics. American Mathematical Society, Providence, RI, second edition, 2001.

[53] M. A. Lopez and S. Reisner. A special case of Mahler's conjecture. Discrete 83 Computational Geometry, 20(2):163-177, 1998.

[54] E. Lutwak, D. Yang, and G. Zhang. Lp affine isoperimetric inequalities. J. Differential Geom, 56(1):111-132, 2000.

[55] E. Lutwak, D. Yang, and G. Zhang. Orlicz centroid bodies. Journal of Differential Geometry, 84(2):365-387, 2010.

[56] E. Lutwak and G. Zhang. Blaschke-Santaló inequalities. J. Differential Geom, 47(1):1-16, 1997.

[57] M Madiman, J Melbourne, and P Xu. Forward and reverse entropy power inequalities in convex geometry. In Convexity and Concentration, pages 427485. Springer, 2017.

[58] K. Mahler. Ein minimalproblem für konvexe polygone. Mathematica (Zutphen) $B, 7: 118-127,1939$. 
[59] M. W. Meckes. Sylvester's problem for symmetric convex bodies and related problems. Monatshefte für Mathematik, 145(4):307-319, 2005.

[60] J. Melbourne. Rearrangement and Prékopa-Leindler Type Inequalities. In High Dimensional Probability VIII, pages 71-97. Springer, 2019.

[61] M. Meyer. Une caractérisation volumique de certains espaces normés de dimension finie. Israel Journal of Mathematics, 55(3):317-326, 1986.

[62] M. Meyer and A. Pajor. On the Blaschke-Santaló inequality. Arch. Math. (Basel), 55(1):82-93, 1990.

[63] M. Meyer and S. Reisner. Shadow systems and volumes of polar convex bodies. Mathematika, 53(1):129-148 (2007), 2006.

[64] M. Meyer and S. Reisner. On the volume product of polygons. In Abhandlungen aus dem Mathematischen Seminar der Universität Hamburg, volume 81, pages 93-100. Springer, 2011.

[65] V. Milman and L. Rotem. Mixed integrals and related inequalities. J. Funct. Anal., 264(2):570-604, 2013.

[66] F. Nazarov. The hörmander proof of the Bourgain-Milman theorem. In Geometric aspects of functional analysis, pages 335-343. Springer, 2012.

[67] F. Nazarov, F. Petrov, D. Ryabogin, and A. Zvavitch. A remark on the Mahler conjecture: local minimality of the unit cube. Duke Mathematical Journal, 154(3):419-430, 2010.

[68] G. Paouris and P. Pivovarov. A probabilistic take on isoperimetric-type inequalities. Adv. Math., 230(3):1402-1422, 2012.

[69] G. Paouris and P. Pivovarov. Randomized isoperimetric inequalities. In Convexity and concentration, volume 161 of IMA Vol. Math. Appl., pages 391-425. Springer, New York, 2017. 
[70] C. M. Petty. Centroid surfaces. Pacific Journal of Mathematics, 11(4):15351547, 1961.

[71] C. M. Petty. Isoperimetric problems. Proceedings of the Conference on Convexity and Combinatorial Geometry (Univ. Oklahoma, Norman, Okla.), pages 26-41, 1971.

[72] R. Pfiefer. The extrema of geometric mean values. Proquest llc. Ann Arbor, MI, 1982.

[73] G. Pisier. The volume of convex bodies and Banach space geometry, volume 94. Cambridge University Press, 1999.

[74] P. Pivovarov and J. Rebollo Bueno. A stochastic Prékopa-Leindler inequality for log-concave functions. Communications in Contemporary Mathematics, 2020. Accepted for publication.

[75] A. Prékopa. Logarithmic concave measures with application to stochastic programming. Acta Sci. Math. (Szeged), 32:301-316, 1971.

[76] A. Prékopa. On logarithmic concave measures and functions. Acta Sci. Math. (Szeged), 34:335-343, 1973.

[77] J. Rebollo Bueno. Stochastic reverse isoperimetric inequalities in the plane. Submitted. https://sites.google.com/view/jesrebbue/research.

[78] S. Reisner. Zonoids with minimal volume-product. Mathematische Zeitschrift, 192(3):339-346, 1986.

[79] S. Reisner, C. Schütt, and E. M. Werner. Mahler's conjecture and curvature. International Mathematics Research Notices, 2012(1):1-16, 2012.

[80] Y. Rinott. On convexity of measures. Ann. Probability, 4(6):1020-1026, 1976.

[81] C. A. Rogers and G. C. Shephard. Some extremal problems for convex bodies. Mathematika, 5:93-102, 1958. 
[82] C.A. Rogers. A single integral inequality. Journal of the London Mathematical Society, 1(1):102-108, 1957.

[83] M. Roysdon and S. Xing. On $L_{p}$-Brunn-Minkowski type and $L_{p}$-isoperimetric type inequalities for general measures. https://arxiv.org/abs/2004. 09737.

[84] J. Saint-Raymond. On the volume of symmetrical convex bodies. Tutorial of Analysis, 81, 1980.

[85] L. A Santaló. Un invariante afin para los cuerpos convexos del espacio de $n$ dimensiones. Portugaliae Mathematica, 8(4):155-161, 1949.

[86] C. Saroglou. Characterizations of extremals for some functionals on convex bodies. Canadian Journal of Mathematics, 62(6):1404-1418, 2010.

[87] C. Saroglou. Shadow systems: remarks and extensions. Archiv der Mathematik, 100(4):389-399, 2013.

[88] R. Schneider. Convex bodies: the Brunn-Minkowski theory, volume 151 of Encyclopedia of Mathematics and its Applications. Cambridge University Press, Cambridge, expanded edition, 2014.

[89] G. C. Shephard. Shadow systems of convex sets. Israel Journal of Mathematics, 2(4):229-236, 1964.

[90] B. Simon. Convexity, volume 187 of Cambridge Tracts in Mathematics. Cambridge University Press, Cambridge, 2011. An analytic viewpoint.

[91] A. Stancu. Two volume product inequalities and their applications. Canadian Mathematical Bulletin, 52(3):464-472, 2009.

[92] J. Sylvester. Question 1491. The Educational Times, April 1864.

[93] M. Weberndorfer. Shadow systems of asymmetric $L_{p}$ zonotopes. Advances in Mathematics, 240:613-635, 2013. 


\section{VITA}

Jesus Rebollo Bueno is from Sevilla, Spain. He received at the Universidad de Sevilla a Bachelor of Science in Mathematics at Universidad de Sevilla in 2013, a Master's in Advance Mathematics in 2014, both under the supervision of Professor Rafael Villa Caro, and a Master's in Mathematics education in 2015. In the same year he started his PhD program at the University of Missouri under the supervision of Professor Peter Pivovarov. 